\title{
Fibrations with noncommutative fibers
}

\author{
Siegfried Echterhoff, Ryszard Nest, and Hervé Oyono-Oyono
}

\begin{abstract}
We study an analogue of fibrations of topological spaces with the homotopy lifting property in the setting of $\mathrm{C}^{*}$-algebra bundles. We then derive an analogue of the Leray-Serre spectral sequence to compute the K-theory of the fibration in terms of the cohomology of the base and the K-theory of the fibres. We present many examples which show that fibrations with noncommutative fibres appear in abundance in nature.
\end{abstract}

Mathematics Subject Classification (2000). 19K35, 46L55, 46L80, 46L85; 14DXX, 46L25, 58B34, 81R60, 81T30.

Keywords. Noncommutative fibrations, noncommutative principal torus bundles, K-theory, Leray-Serre spectral sequence.

\section{Contents}

1 Some preliminaries . . . . . . . . . . . . . . . . . 380

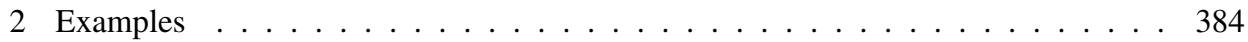

3 The group bundle corresponding to an $h$-fibration $\ldots \ldots \ldots \ldots$

4 The Leray-Serre spectral sequence . . . . . . . . . . . . . . . . . . . . . . 390

5 Applications to noncommutative torus bundles . . . . . . . . . . . . . . 407

\section{Introduction}

In recent years the study of the topological properties of $\mathrm{C}^{*}$-algebra bundles plays a more and more prominent rôle in the field of operator algebras. The main reason for this is two-fold: on one side there are many important examples of $\mathrm{C}^{*}$-algebras which do come with a canonical bundle structure. On the other side, the study of $\mathrm{C}^{*}$-algebra bundles over a locally compact Hausdorff base space $X$ is the natural next step in classification theory, after the far reaching results which have been obtained in the classification of simple $\mathrm{C}^{*}$-algebras. To fix notation, by a $C^{*}$-algebra bundle $A(X)$ over $X$ we shall simply mean a $C_{0}(X)$-algebra in the sense of Kasparov (see [17]): it is a $C^{*}$-algebra $A$ together with a non-degenerate ${ }^{*}$-homomorphism

$$
\Phi: C_{0}(X) \rightarrow Z M(A),
$$


where $Z M(A)$ denotes the center of the multiplier algebra $M(A)$ of $A$. For such $C_{0}(X)$-algebra $A$, the fibre over $x \in X$ is then $A_{x}=A / I_{x}$, where

$$
I_{x}=\left\{\Phi(f) \cdot a \mid a \in A \text { and } f \in C_{0}(X) \text { such that } f(x)=0\right\},
$$

and the canonical quotient map $q_{x}: A \rightarrow A_{x}$ is called the evaluation map at $x$. We shall often write $A(X)$ to indicate the given $C_{0}(X)$-structure of $A$. We shall recall the basic constructions and properties of $C_{0}(X)$-algebras in the preliminary section below. We refer to [10] for further notation concerning $C_{0}(X)$-algebras.

The main problem when studying bundles from the topological point of view is to provide good topological invariants which help to understand the local and global structure of the bundles. A good example is given by the class of separable continuous-trace $\mathrm{C}^{*}$-algebras, which are, up to Morita equivalence, just the section algebras of locally trivial bundles over $X$ with fibres the compact operators $\mathcal{K} \cong$ $\mathcal{K}\left(l^{2}(\mathbb{N})\right)$. Using the standard classification of fibre bundles, these algebras (or rather the underlying bundle structure) are classified up to Morita equivalence by a corresponding Dixmier-Douady class in $\check{H}^{3}(X, \mathbb{Z})$. Another interesting class of examples are the noncommutative principle torus bundles, which have been studied by the authors in [10]. A basic example of a noncommutative principal 2-torus bundle is given by the $\mathrm{C}^{*}$-algebra $C^{*}(H)$ of the discrete rank 3 Heisenberg group $H$, which has a canonical structure of a $\mathrm{C}^{*}$-algebra bundle over the circle $\mathbb{W}$ where the fibre $A_{z}$ over $z \in \mathbb{T}$ is the noncommutative 2-torus $A_{\theta}$ if $z=e^{2 \pi i \theta}$. This shows in particular, that such bundles are in general far away from being section algebras of locally trivial $\mathrm{C}^{*}$-algebra bundles (but see [10], §2, for a classification based on classical methods).

The main purpose of [10] was the study of the K-theoretic properties of the principle noncommutative $\mathbb{T}^{n}$-bundles after forgetting the $\mathbb{T}^{n}$-actions. Using Kasparov's $\mathcal{R K K}(X ; \cdot, \cdot)$-theory as the version of $\mathrm{K}$-theory which is probably most adapted to the study $\mathrm{C}^{*}$-algebra bundles, we show in [10], Corollary 3.4, that the noncommutative $\mathbb{T}^{n}$-bundles are always locally $\mathcal{R K K}$-trivial, which means that for each $x \in X$ there exists a neighbourhood $U$ of $x$ such that the restriction $A(U)$ of $A(X)$ to $U$ is $\mathcal{R K K}(U ; \cdot, \cdot)$-equivalent to $C_{0}\left(U \times \mathbb{T}^{n}\right)$. As usual, the global picture is much more difficult. Using the local $\mathcal{R} K K$-triviality we show in [10] that to each noncommutative torus bundle $A(X)$ we may associate a corresponding bundle of $\mathrm{K}$-theory groups which comes equipped with a canonical action of the fundamental group $\pi_{1}(X)$ of the base $X$. Using this associated group bundle allows us to obtain at least a partial classification result up to $\mathcal{R K K}$-equivalence (see [10], Theorem 7.5).

In this article we want to extend the studies of [10] from a more general perspective. Indeed, we are interested in $\mathrm{C}^{*}$-algebra bundles which are noncommutative analogues of classical fibrations in topology which satisfy certain weak versions of the homotopy lifting property. Indeed, the important point implied by the homotopy lifting property in classical topology is that for any fibration $q: Y \rightarrow X$ with this property, the space $Y$ looks, in a topological sense, locally like a product space $U \times F$. The phrase "in a 
topological sense" means that any homotopy invariant (co-)homology theory cannot differentiate between $p^{-1}(U)$ and $U \times F$.

Since it seems to be impossible to rephrase the homotopy lifting property in the noncommutative setting, we shall give a definition of this property in dependence of a given (co-)homology theory on the category of $\mathrm{C}^{*}$-algebras. For example, a (section algebra of a) $\mathrm{C}^{*}$-algebra bundle $A(X)$ over $X$ is called a $\mathrm{K}$-fibration if for any positive integer $p$, for any $p$-simplex $\Delta^{p}$ and for any continuous map $f: \Delta^{p} \rightarrow X$ the pullback $f^{*} A\left(\Delta^{p}\right)$ of $A(X)$ via $f$ is $\mathrm{K}$-theoretically trivial in the sense that the evaluation homomorphism

$$
q_{v}: f^{*} A\left(\Delta^{p}\right) \rightarrow A_{f(v)}
$$

induces an isomorphism of K-theory groups $\mathrm{K}_{i}\left(f^{*} A\left(\Delta^{p}\right)\right) \cong \mathrm{K}_{i}\left(A_{f(v)}\right)$ for all $v \in \Delta^{p}$. In a similar way we can define KK-fibrations, $\mathcal{R K K}$-fibrations or $h$-fibrations, when $\left(h_{n}\right)_{n \in \mathbb{Z}}$ (resp. $\left(h^{n}\right)_{n \in \mathbb{Z})}$ is any given (co-)homology theory on a suitable category of $\mathrm{C}^{*}$-algebras. In case of K-theory, the strongest notion will be that of an $\mathcal{R K K}$-fibration (which implies that such bundles are automatically KK- and $\mathrm{K}$-fibrations) and we shall indicate that there exist many natural examples of such fibrations. For instance, the principle noncommutative torus bundles of [10] are always $\mathcal{R K K}$-fibrations.

The main result of this article will be the proof of a noncommutative analogue of the Leray-Serre spectral sequence for general $h$-fibrations. Indeed, if $\left(h_{n}\right)_{n \in \mathbb{Z}}$ (resp. $\left(h^{n}\right)_{n \in \mathbb{Z}}$ ) is any given (co-)homology theory on a suitable category of $\mathrm{C}^{*}$-algebras, and if $A(X)$ is an $h$-fibration over the geometric realisation of a simplicial complex $X$, we show that we can associate to $A$ the group bundle $\mathscr{H}=\left\{h_{q}\left(A_{x}\right) \mid x \in\right.$ $X\}$ which carries a canonical action of $\pi_{1}(X)$. The Leray-Serre spectral sequence for $A(X)$ then converges to $h(A(X))$ and has (co-)homology groups $H^{p}\left(X, \mathscr{H}_{q}\right)$ as $E_{2}$-terms.

Thus, at least in principle we can use the spectral sequence for computation of the $\mathrm{K}$-theory groups of any $\mathrm{K}$-fibration $A(X)$. In particular this applies to the principal noncommutative $\mathbb{T}^{n}$-bundles as studied in [10]. The spectral sequence also serves as an obstruction for $\mathcal{R K K}$-equivalence of two bundles $A(X)$ and $B(X)$ - any such equivalence induces an isomorphism between the respective spectral sequences. It is certainly an interesting question to what extend the converse might hold, at least in case where $A(X)$ and $B(X)$ are $\mathcal{R K K}$-fibrations (or locally $\mathcal{R K K}$-trivial). In a final section we apply the spectral sequence to the study of the noncommutative torus bundles of [10] and show that it gives the missing tool for deciding which noncommutative torus bundles are globally $\mathcal{R K K}$-trivial. We further give an explicit computation of the spectral sequences in the case of noncommutative 2-torus bundles over $\mathbb{T}^{2}$. The results show that there are noncommutative principle torus bundles with isomorphic spectral sequences for which we do not know at this point whether they are $\mathcal{R K K}$-equivalent. 


\section{Some preliminaries}

1.1. Homology theories on $\mathbf{C}^{*}$-algebras. Let $\boldsymbol{C}_{\text {all }}$ denote the category of all $\mathrm{C}^{*}$ algebras with *-homomorphisms as morphisms. By a good subcategory of $\mathcal{C}_{\text {all }}$ we mean any subcategory $\ell$ of $\ell_{\text {all }}$ with $\mathbb{C} \in \ell$ and which is closed under taking ideals, quotients, extensions and suspension in the sense that if $A \in \mathrm{Ob}(\mathcal{C})$, then $S A:=$ $C_{0}(\mathbb{R}, A) \in \mathrm{Ob}(\mathcal{C})$. Moreover, for simplicity, we shall assume that $\operatorname{Mor} \mathcal{C}(A, B)=$

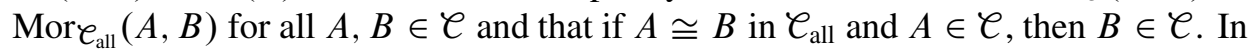
many cases considered below, the above assumption on the morphisms could probably be weakened to the assumptions given in [2], 21.1, but we do not want to bother with this extra generality. Standard examples of good subcategories of $\mathcal{C}_{\text {all }}$ are given by the category $\ell_{\text {sep }}$ of separable $C^{*}$-algebras or the category $\ell_{\text {nuc }}$ of nuclear $\mathrm{C}^{*}$-algebras. Following [2], 21.1, we define:

Definition 1.1. A homology theory on a good subcategory $\varphi$ of $\ell_{\text {all }}$ is a sequence $\left\{h_{n}\right\}_{n \in \mathbb{Z}}$ of covariant functors $h_{n}$ from $\ell$ to the category Ab of abelian groups satisfying the following axioms:

(H) If $f_{0}, f_{1}: A \rightarrow B$ are homotopic, then $f_{0, *}=f_{1, *}: h_{n}(A) \rightarrow h_{n}(B)$ for all $n \in \mathbb{Z}$.

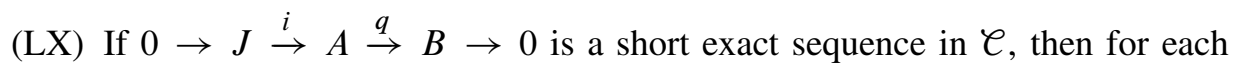
$n \in \mathbb{Z}$ there are connecting maps $\partial_{n}: h_{n}(B) \rightarrow h_{n-1}(J)$, natural with respect to morphisms of short exact sequences, making exact the following long sequence

$$
\cdots \stackrel{\partial_{n+1}}{\longrightarrow} h_{n}(J) \stackrel{i_{*}}{\longrightarrow} h_{n}(A) \stackrel{q_{*}}{\longrightarrow} h_{n}(B) \stackrel{\partial_{n}}{\longrightarrow} h_{n-1}(J) \stackrel{i_{*}}{\rightarrow} \cdots .
$$

Similarly, we define a cohomology theory on a good subcategory as a sequence $\left\{h^{n}\right\}_{n \in \mathbb{Z}}$ of contravariant functors $h^{n}: € \rightarrow$ Ab which satisfy the obvious reversed axioms (e.g., see [2], 21.1).

Remark 1.2. (1) It follows from these axioms that all $h_{n}: \bigodot \rightarrow$ Ab are additive in the sense that

$$
h_{n}\left(A_{1} \oplus A_{2}\right)=h_{n}\left(A_{1}\right) \oplus h_{n}\left(A_{2}\right)
$$

and that $h_{n}(A)=\{0\}$ if $A$ is a contractible $C^{*}$-algebra. Since $C A:=C((-\infty, \infty], A)$ is contractible, it follows from (LX) applied to the short exact sequence

$$
0 \rightarrow S A \rightarrow C A \rightarrow A \rightarrow 0
$$

that $h_{n+1}(A)=h_{n}(S A)$ (resp. $\left.h^{n-1}(A)=h^{n}(S A)\right)$ for all $A \in \mathcal{C}$.

(2) A covariant (resp. contravariant) functor $F: \mathcal{C}_{\text {sep }} \rightarrow$ Ab is called stable if

$$
i_{p}: A \rightarrow A \otimes \mathcal{K}, \quad i_{p}(a)=a \otimes p
$$


induces an isomorphism $i_{p, *}: F(A) \stackrel{\cong}{\longrightarrow} F(A \otimes \mathcal{K})\left(\right.$ resp. $\left.i_{p}^{*}: F(A \otimes \mathcal{K}) \stackrel{\cong}{\longrightarrow} F(A)\right)$ for every one-dimensional projection $p \in \mathcal{K}$. It is shown in [2], Corollary 22.3.1, (the result is originally due to Cuntz [5]) that every stable (co-)homology theory $\left\{h_{n}\right\}$ (resp. $\left\{h^{n}\right\}$ ) on $\mathcal{C}_{\text {sep }}$ satisfies Bott-periodicity $h_{n+2}(A)=h_{n}\left(S^{2} A\right) \cong h_{n}(A)$ (resp. $h^{n+2}(A) \cong h^{n}(A)$ ). Hence, every stable (co-)homology theory on $\ell_{\text {sep }}$ is $\mathbb{Z} / 2 \mathbb{Z}$-graded and the long exact sequence (LX) then becomes a cyclic six-term exact sequence.

(3) A homology theory $\left\{h_{n}\right\}$ is called $\sigma$-additive (resp. $\sigma$-multiplicative) if $h_{n}(A)=\bigoplus_{i \in I} h_{n}\left(A_{i}\right)\left(\right.$ resp. $\left.h_{n}(A)=\prod_{i \in I} h_{n}\left(A_{i}\right)\right)$ whenever $A \in \mathcal{C}$ is a countable direct sum of objects $A_{i} \in \mathcal{C}, i \in I$, and similarly for cohomology theories.

(4) The main example of a homology theory on $\mathcal{C}_{\text {all }}$ (or any good subcategory $\mathcal{C}$ of $\left.\mathcal{C}_{\text {all }}\right)$ is given by $\mathrm{K}$-theory, and $\mathrm{K}$-homology serves as the main example for a cohomology theory on $\mathcal{C}_{\text {all }}$. Note that $\mathrm{K}$-theory is $\sigma$-additive and $\mathrm{K}$-homology is $\sigma$-multiplicative.

Assume now that $\mathcal{C}$ is a good subcategory of $\mathcal{C}_{\text {all }}$ and suppose that $A(X) \in \mathcal{C}$ is a $C_{0}(X)$-algebra. In what follows we write $\Delta^{p}=<v_{0}, \ldots, v_{p}>$ for the standard $p$ simplex with vertices $v_{0}, \ldots, v_{p}$. It follows from the properties of a good subcategory of $\mathcal{C}_{\text {all }}$ that if $A \in \mathcal{C}$ and $f: \Delta^{p} \rightarrow X$ is any continuous map, then $f^{*} A=\left(C\left(\Delta^{p}\right) \otimes\right.$ $A) / I_{f}$, with $I_{f}$ is a suitable ideal in $C\left(\Delta^{p}\right) \otimes A$, is again an object in $C$. In particular, all fibers $A_{x}$ for $x \in X$ are in $\mathcal{C}$. The following definition is motivated by the notation and results presented in [8], Chapter I:

Definition 1.3. Suppose that $\mathcal{C}$ is a good subcategory of $\mathcal{C}_{\text {all }}$ and that $\left\{h_{n}\right\}$ is a homology theory on $\mathcal{C}$ (resp. $\left\{h^{n}\right\}$ is a cohomology theory on $\mathcal{C}$ ). Suppose further that $A=A(X)$ is a $C_{0}(X)$-algebra in $\mathcal{C}$. Then

(i) $A(X)$ is called an $h$-fibration if for all continuous maps $f: \Delta^{p} \rightarrow X$ and for every point $v \in \Delta^{p}$, the quotient map $q_{v}: f^{*} A \rightarrow A_{f(v)}$ induces an isomorphism $q_{v, *}: h_{n}\left(f^{*} A\right) \rightarrow h_{n}\left(A_{f(v)}\right)$ (resp. $q_{v}^{*}: h^{n}\left(A_{f(v)}\right) \rightarrow h^{n}\left(f^{*} A\right)$ ).

(ii) If $\mathcal{C}=\mathcal{C}_{\text {sep }}$, then $A(X)$ is called a KK-fibration, if for all continuous maps $f: \Delta^{p} \rightarrow X$ and for every element $v \in \Delta^{p}$ the quotient map $q_{v}: f^{*} A \rightarrow A_{f(v)}$ is a $\mathrm{KK}$-equivalence.

(iii) If $\mathcal{C}=\mathcal{C}_{\text {sep }}$, then $A(X)$ is called an $\mathcal{R K K}$-fibration, if $f^{*} A$ is $\mathcal{R K K}\left(\Delta^{p} ; \cdot, \cdot\right)$ equivalent to $C\left(\Delta^{p}, A_{f(v)}\right)$ for any continuous map $f: \Delta^{p} \rightarrow X$ and for any element $v$ of $\Delta^{p}$.

Remark 1.4. (1) Any $\mathcal{R K K}$-fibration is a KK-fibration. This follows from the fact that if $x \in \mathcal{R K K}\left(\Delta^{p} ; C\left(\Delta^{p}, A_{f(v)}\right), f^{*} A\right)$ is an $\mathcal{R K K}$-equivalence, then we get the 
following commutative diagram in $\mathrm{KK}$ :

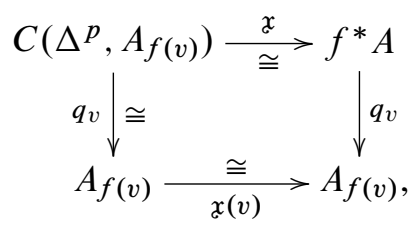

where all arrows except of the right vertical one are known to be isomorphisms in KK. But then all arrows are KK-equivalences. We shall formulate below a partial converse of this easy observation, which follows from a result of Dadarlat.

(2) It is a direct consequence of [2], Corollary 22.3.1, that if $A(X)$ is a KK-fibration, then $A(X)$ is an $h$-fibration for any stable (co-)homology theory $\left\{h_{n}\right\}$ (resp. $\left\{h^{n}\right\}$ ) on $\varphi_{\text {sep. }}$

(3) Every locally trivial $\mathrm{C}^{*}$-algebra bundle $A(X)$ is an $\mathcal{R K K}$-fibration. This follows from the fact that a pull-back of a locally trivial $\mathrm{C}^{*}$-algebra bundle is again locally trivial, and that any locally trivial bundle over a contractible space is trivial (e.g., see [16]).

(4) All noncommutative principal $n$-tori as considered in [10] are $\mathcal{R K K}$-fibrations. This follows from [10], Proposition 3.1.

(5) Being an $h$-fibration (resp. KK-fibration, resp. $\mathcal{R K K}$-fibration) is preserved by taking pull-backs inside $\mathcal{C}$. This follows from the fact that if $A$ is a $C_{0}(X)$-algebra in $\zeta$ and $g: Y \rightarrow X$ is any continuous map such that $g^{*}(A) \in \mathcal{C}$, and if $f: \Delta^{p} \rightarrow Y$ is any continuous map, then $f^{*}\left(g^{*}(A)\right)=(g \circ f)^{*}(A)$, and hence evaluation at any vertex induces isomorphisms in $h$-theory.

(6) Being a KK-fibration is preserved under taking maximal tensor products with arbitrary separable $\mathrm{C}^{*}$-algebras and by minimal tensor products with separable exact $\mathrm{C}^{*}$-algebras. This follows from the fact that taking maximal or minimal tensor products of a KK-equivalence $x \in \mathrm{KK}_{0}(C, D)$ with a fixed $\mathrm{C}^{*}$-algebra $B$ gives a KK-equivalence

$$
x \otimes B \in \mathrm{KK}_{0}\left(C \otimes_{(\max )} B, D \otimes_{(\max )} B\right) .
$$

Similar statements hold for $\mathcal{R K K}$-fibrations.

In what follows next we want to show that in many situations being a KK-fibration is actually equivalent to being an $\mathcal{R K K}$-fibration. Recall that a $\mathrm{C}^{*}$-algebra bundle (i.e., a $C_{0}(X)$-algebra) $A(X)$ is called a continuous $C^{*}$-algebra bundle if for all $a \in A$ the map $x \mapsto\left\|a_{x}\right\|$ is a continuous function on $X$. We need the following deep theorem of Dadarlat (see [6], Theorem 1.1).

Theorem 1.5. Let $X$ be a compact metrizable finite dimensional space and let $A(X)$ and $B(X)$ be separable nuclear continuous $C^{*}$-algebra bundles over $X$. Suppose 
further that $\sigma \in \mathcal{R K K}(X ; A(X), B(X))$ is such that $\sigma(x) \in \mathrm{KK}\left(A_{x}, B_{x}\right)$ is invertible for all $x \in X$. Then $\sigma$ is invertible in $\mathcal{R K K}(X ; A(X), B(X))$.

As a direct corollary we get the partial converse to the observation made in item (2) of Remark 1.4:

Corollary 1.6. Suppose that $A(X)$ is a separable nuclear continuous $C^{*}$-algebra bundle over some locally compact space $X$. Then $A(X)$ is a KK-fibration if and only if it is an RKK-fibration.

Proof. Since every $\mathcal{R K K}$-fibration is a KK-fibration by item (1) of Remark 1.4 we only have to show the converse. Write $\Delta:=\Delta^{p}$ and let $f: \Delta \rightarrow X$ be any continuous map. Since $A$ is a KK-fibration, there exists the inverse $q_{v}^{-1} \in \operatorname{KK}\left(A_{f(v)}, f^{*} A\right)$ of the evaluation map $q_{v}$. Consider the image of $q_{v}^{-1}$ under the composition of maps

$$
\begin{aligned}
\mathrm{KK}\left(A_{f(v)}, f^{*} A\right) \stackrel{\sigma_{\Delta, C(\Delta)}}{\longrightarrow} \mathcal{R K K}\left(\Delta ; C(\Delta) \otimes A_{f(v)}, C(\Delta) \otimes f^{*} A\right) \\
\stackrel{\mu_{*}}{\longrightarrow} \mathcal{R K K}\left(\Delta ; C\left(\Delta, A_{f(v)}\right), f^{*} A\right),
\end{aligned}
$$

where $\mu: C(\Delta) \otimes f^{*} A \rightarrow f^{*} A ; \mu(g \otimes a)=g \cdot a$ is the multiplication homomorphism. If we evaluate this class at a point $w \in \Delta$, we obtain the class $\left(q_{v}^{-1}\right) \otimes q_{w} \in \mathrm{KK}\left(A_{f(v)}, A_{f(w)}\right)$, which is invertible since $A$ is a KK-fibration. Hence the result follows from Dadarlat's theorem.

Another interesting problem is the relation between locally $\mathcal{R K K}$-triviality, which was discussed in [10] in connection with noncommutative torus bundles and the $\mathcal{R K K}$-fibrations considered here. Let us recall that a $\mathrm{C}^{*}$-algebra bundle $A(X)$ is called locally $\mathcal{R K K}$-trivial, if for every $x \in X$ there exists a neighbourhood $V$ of $x$ such that the restriction $A(V)$ of $A$ to $V$ is $\mathcal{R K K}(V ; \cdot, \cdot)$-equivalent to $C_{0}\left(V, A_{x}\right)$. We have seen in [10] that all principal noncommutative torus bundles are locally $\mathcal{R K K}$-trivial. The proof of the following proposition is then straightforward.

Proposition 1.7. Suppose that $X$ is locally euclidean, i.e., every $x \in X$ has a neighbourhood $U$ which is homeomorphic to an open ball in some $\mathbb{R}^{n}$. Then, if $A(X)$ is an $\mathcal{R K K}$-fibration it follows that $A(X)$ is locally $\mathcal{R K K}$-trivial.

A bit surprisingly, the converse of the above proposition seems to be much more complicated. We shall obtain it later as a corollary of another remarkable theorem of Dadarlat (see [6], Theorem 2.5), which states that every separable and nuclear continuous $\mathrm{C}^{*}$-algebra bundle over some compact metrizable space $X$ is $\mathcal{R K K}(X ; \cdot, \cdot)$-equivalent to a continuous bundle of simple Kirchberg algebras, i.e., each fibre is a separable nuclear purely infinite $\mathrm{C}^{*}$-algebra. As a direct consequence we obtain 
Proposition 1.8. Suppose that $A(X)$ is a separable nuclear continuous $C^{*}$-algebra bundle over the compact metrizable finite dimensional space $X$ such that $A(X)$ is locally $\mathcal{R K K}$-trivial. Then $A(X)$ is $\mathcal{R K K}$-equivalent to a locally trivial bundle of stable Kirchberg algebras.

Proof. By Dadarlat's theorem, we may assume that $A(X)$ is a $\mathrm{C}^{*}$-algebra bundle of simple Kirchberg algebras, and by stabilizing this bundle, we may assume that all fibers are stable. If $A(X)$ is locally $\mathcal{R K K}$-trivial, we can find for each $x \in X$ a compact neighbourhood $V_{x}$ such that $A\left(V_{x}\right) \sim_{\mathcal{R K K}} C\left(V_{x}, A_{x}\right)$. It is then a consequence of [6], Theorem 2.7, that this equivalence is actually realized by an isomorphism $A\left(V_{x}\right) \cong C\left(V_{x}, A_{x}\right)$ of $\mathrm{C}^{*}$-algebra bundles over $V_{x}$.

As a corollary we get

Corollary 1.9. If $A(X)$ is a separable nuclear continuous field of $C^{*}$-algebras over a locally compact space $X$. If $A(X)$ is locally $\mathcal{R K K}$-trivial, then $A(X)$ is an $\mathcal{R K K}$ fibration.

Proof. If $f: \Delta^{p} \rightarrow A(X)$ is any continuous map, the pull-back $f^{*} A\left(\Delta^{p}\right)$ satisfies all requirements of the above proposition. Since $\Delta^{p}$ is contractible, every locally trivial bundle over $\Delta^{p}$ is trivial. Thus it follows from the proposition that $f^{*} A\left(\Delta^{p}\right)$ is $\mathcal{R K K}$-equivalent to a trivial bundle.

\section{Examples}

In this section we want to show that K-fibrations and KK-fibrations do appear quite often in nature. We already mentioned above that all locally trivial $\mathrm{C}^{*}$-algebra bundles are $\mathcal{R K K}$-fibrations. Since being an $\mathcal{R K K}$-fibration is stable under $C_{0}(X)$-linear Morita equivalence, this implies also that all continuous-trace $C^{*}$-algebras with spectrum $X$ are $\mathcal{R} K K$-fibrations. Although these classes of $\mathrm{C}^{*}$-algebra bundles are certainly interesting, it would probably not give enough motivation for a general study of fibrations as we do in this article.

A class of interesting algebras which are, in general, far away from being locally trivial bundles of $\mathrm{C}^{*}$-algebras are the noncommutative principal torus bundles as studied in [10], and we already pointed out that all of them are $\mathcal{R K K}$-fibrations. Recall that the principal noncommutative torus bundles are, by definition, crossed products of the form $C_{0}(X, \mathcal{K}) \rtimes \mathbb{Z}^{n}$, where $\mathbb{Z}^{n}$ acts fibrewise on the trivial bundle $C_{0}(X, \mathcal{K})$. We shall now see that, with the help of the Baum-Connes conjecture, one can construct many other examples of $\mathcal{R K K}-, \mathrm{KK}-$, or K-fibrations via a similar crossed product construction. 
Suppose that $A$ is a $\mathrm{C}^{*}$-algebra bundle and $\alpha: G \rightarrow \operatorname{Aut}(A)$ is any $C_{0}(X)$-linear action of the locally compact group $G$ on $A$, i.e., we have

$$
\alpha_{s}(f \cdot a)=f \cdot \alpha_{s}(a) \text { for all } s \in G, f \in C_{0}(X) \text { and } a \in A .
$$

(We simply write $f \cdot a$ for $\Phi(f) a$ if $\Phi: C_{0}(X) \rightarrow Z M(A)$ is the $C_{0}(X)$-structure map of the bundle.) Then $\alpha$ induces actions $\alpha^{x}: G \rightarrow \operatorname{Aut}\left(A_{x}\right)$ on the fibres $A_{x}$ via $\alpha_{s}^{x}\left(a+I_{x}\right)=\alpha_{s}(a)+I_{x}$. The full and reduced crossed products $A \rtimes_{(r)} G$ have canonical structures as $C_{0}(X)$-algebras via the composition of the given $C_{0}(X)$-structure $\Phi: C_{0}(X) \rightarrow Z M(A)$ of $A$ with the canonical embedding $M(A) \rightarrow M\left(A \rtimes_{(r)} G\right)$. For the full crossed product $A \rtimes G$, the fibre over $x \in X$ is then given by the full crossed product $A_{x} \rtimes G$, which follows from the exactness of full crossed with respect to short exact sequences of $G$-algebras. For the reduced crossed products the situation can be more complicated. However, if $G$ is exact in the sense of Kirchberg and Wassermann (which is true for a large class of groups - see [18]), then the fibre of $A \rtimes_{r} G$ over $x \in X$ is $A_{x} \rtimes_{r} G$.

Note also that if $f: Y \rightarrow X$ is any continuous map, and if $\alpha: G \rightarrow \operatorname{Aut}(A)$ is a $C_{0}(X)$-linear action of $G$ on $A$, then we get a $C_{0}(Y)$-linear pull-back action $f^{*}(\alpha): G \rightarrow \operatorname{Aut}\left(f^{*} A\right)$ given on elementary tensors $g \otimes a \in f^{*} A=C_{0}(Y) \otimes C_{0}(X)$ $A$ by the formula

$$
f^{*}(\alpha)_{s}(g \otimes a):=g \otimes \alpha_{s}(a) .
$$

It is then easily checked (e.g., see [11]), that $f^{*} A \rtimes G \cong f^{*}(A \rtimes G)$ as $C_{0}(Y)$ algebras and $f^{*} A \rtimes_{r} G \cong f^{*}\left(A \rtimes_{r} G\right)$ if $G$ is exact.

In what follows next, we want to give some conditions which imply that the $C_{0}(X)$-algebras $A \rtimes G$ and $A \rtimes_{r} G$ are either $\mathrm{K}_{*}$-fibrations, KK-fibrations, or even $\mathcal{R}$ KK-fibrations. As the basic tool for this we shall use the Baum-Connes conjecture for $G$. Recall that for any $G$-algebra $A$, the topological K-theory of $G$ with coefficient $A$ is defined as

$$
\mathrm{K}_{*}^{\mathrm{top}}(G ; A)=\lim _{Z} \mathrm{KK}_{*}^{G}\left(C_{0}(Z), A\right),
$$

where $Z$ runs through the $G$-compact subspaces of a universal proper $G$-space $\mathcal{E}(G)$. In [1], Baum, Connes and Higson constructed an assembly map

$$
\mu_{A}: \mathrm{K}_{*}^{\mathrm{top}}(G ; A) \rightarrow \mathrm{K}_{*}\left(A \rtimes_{r} G\right)
$$

and they conjectured that this map should always be an isomorphism of groups. Although this conjecture turned out to be false in general (e.g., see [15]), the conjecture has been shown to be true for very large classes of groups including all amenable and, more generally, a-T-menable groups (see [14]). In what follows, if $A$ is a fixed $G$ algebra, we shall say that $G$ satisfies $B C$ for $A$ if the map is an isomorphism for this special $G$-algebra $A$.

A-T-menable groups satisfy in fact a stronger version of the Baum-Connes conjecture, which can be stated as follows. Recall that a $G$-algebra $D$ is said to be a proper 
$G$-algebra if $D$ is a $C_{0}(Z)$-algebra for some proper $G$-space $Z$ in such a way that the structure map $\Phi: C_{0}(Z) \rightarrow Z M(D)$ is $G$-equivariant. A group $G$ is said to have a $\gamma$-element if there exists an element $\gamma_{G} \in \mathrm{KK}_{0}^{G}(\mathbb{C}, \mathbb{C})$ and a proper $G$-algebra $D$ such that $\gamma_{G}$ can be written as a Kasparov product $\beta \otimes_{D} \delta$ for some $\beta \in \mathrm{KK}_{0}^{G}(\mathbb{C}, D)$ and $\delta \in \mathrm{KK}_{0}^{G}(D, \mathbb{C})$, and such that the restriction $\operatorname{res}_{K}^{G}(\gamma)=1 \in \mathrm{KK}_{0}^{K}(\mathbb{C}, \mathbb{C})$ for all compact subgroups $K$ of $G$. If $G$ has a $\gamma$-element as above, then, by work of Kasparov [17] and Tu [21] (extended in [4], Theorem 1.11, to the weaker notion of a $\gamma$-element used here) the Baum-Connes assembly map is known to be split injective with image $\mu_{A}\left(\mathrm{~K}_{*}^{\text {top }}(G ; A)\right)=\gamma_{G} \cdot \mathrm{K}_{*}\left(A \rtimes_{r} G\right)$. We say that $G$ satisfies the strong Baum-Connes conjecture if $\gamma_{G}=1_{G}$ in $\mathrm{KK}_{0}^{G}(\mathbb{C}, \mathbb{C})$. By the results of Higson and Kasparov in [14], every a-T-menable group satisfies the strong Baum-Connes conjecture. It is clear from the above discussion that every group $G$ which satisfies the strong Baum-Connes conjecture satisfies BC for all $G$-algebras $A$.

Proposition 2.1. Suppose that $A$ and $B$ are $G$-algebras and that $q \in \mathrm{KK}^{G}(A, B)$. Let $j_{G}^{(r)}(q) \in \mathrm{KK}_{0}\left(A \rtimes_{(r)} G, B \rtimes_{(r)} G\right)$ denote the descent of $q$ for the full (resp. reduced) crossed products. For every compact subgroup $K$ of $G$ let

$$
\varphi_{K}: \mathrm{K}_{*}(A \rtimes K) \rightarrow \mathrm{K}_{*}(B \rtimes K) ; \varphi_{K}(x)=x \otimes j_{K}\left(\operatorname{res}_{K}^{G}([q])\right) .
$$

Then the following is true:

(i) If $G$ satisfies $B C$ for $A$ and $B$ and if $\varphi_{K}$ is an isomorphism for every compact subgroup $K$ of $G$, then $\cdot \otimes j_{G}^{r}(q): \mathrm{K}_{*}\left(A \rtimes_{r} G\right) \rightarrow \mathrm{K}_{*}\left(B \rtimes_{r} G\right)$ is an isomorphism.

(ii) If $G$ satisfies the strong Baum-Connes conjecture and if $\varphi_{K}$ is an isomorphism for every compact subgroup $K$ of $G$, then

$$
\cdot \otimes j_{G}^{(r)}(q): \mathrm{K}_{*}\left(A \rtimes_{(r)} G\right) \rightarrow \mathrm{K}_{*}\left(B \rtimes_{(r)} G\right)
$$

is an isomorphism for the full and reduced crossed products.

(iii) If $G$ satisfies the strong Baum-Connes conjecture and if $j_{K}\left(\operatorname{res}_{K}^{G}(q)\right)$ is a KK-equivalence between $A \rtimes K$ and $B \rtimes K$ for all compact subgroups $K$ of $G$, then $j_{G}^{(r)}(q)$ is a KK-equivalence between $A \rtimes_{(r)} G$ and $B \rtimes_{(r)} G$, for the full and reduced crossed products.

Proof. Since $G$ satisfies BC for $A$ and $B$ item (i) follows if we can show that taking Kasparov product with $q$ induces an isomorphism from $\mathrm{K}_{*}^{\text {top }}(G ; A)$ to $\mathrm{K}_{*}^{\text {top }}(G ; B)$. But since all $\varphi_{K}$ are isomorphisms, this follows from [9], Proposition 1.6.

The proof of (ii) is a consequence of (i) and the fact that the strong Baum-Connes conjecture implies the Baum-Connes conjecture for all $G$-algebras and it implies also that $G$ is $\mathrm{K}$-amenable, from which it follows that the regular representation $L: A \rtimes G \rightarrow A \rtimes_{r} G$ induces an isomorphism in K-theory [22]. 
Finally, the proof of (iii) follows from the second part of [20], Proposition 8.5, since under the assumption of the strong Baum-Connes conjecture, the derived crossed products $A \rtimes^{\mathbb{L}} G$ and $B \rtimes^{\mathbb{Z}} G$ of [20], Proposition 8.5, are KK-equivalent to the full and reduced crossed products $A \rtimes_{(r)} G$ and $B \rtimes_{(r)} G$, respectively.

The above proposition now implies the following result.

Proposition 2.2. Suppose that $A=A(X)$ is a separable $C^{*}$-algebra bundle over $X$ and let $\alpha: G \rightarrow \operatorname{Aut}(A)$ be a $C_{0}(X)$-linear action of the second countable locally compact group $G$ on $A(X)$. Assume that for each compact subgroup $K$ of $G$ the $C_{0}(X)$-algebra $A(X) \rtimes K$ is a $\mathrm{K}_{*}$-fibration. Then the following holds:

(i) If $G$ is exact and satisfies $B C$ for $f^{*} A$ for all continuous $f: \Delta^{p} \rightarrow X$, $p=0,1,2, \ldots$ (in particular, if $G$ satisfies $B C$ for all $G$-algebras $B$ ), then the reduced crossed product $A(X) \rtimes_{r} G$ is a $\mathrm{K}_{*}$-fibration.

(ii) If $G$ satisfies the strong Baum-Connes conjecture, then the full crossed product $A(X) \rtimes G$ is a $\mathrm{K}_{*}$-fibration. If, in addition, $G$ is exact, the same is true for the reduced crossed product $A(X) \rtimes_{r} G$.

(iii) If $G$ satisfies the strong Baum-Connes conjecture and if $A(X) \rtimes K$ is a KKfibration for every compact subgroup $K \subseteq G$, then $A(X) \rtimes G$ is a KK-fibration. If, in addition, $G$ is exact, then $A(X) \rtimes_{r} G$ is a KK-fibration, too.

Proof. If $G$ is exact, then $A \rtimes_{r} G$ is a $C_{0}(X)$-algebra with fibres $A_{x} \rtimes_{r} G$ and we have $f^{*} A \rtimes_{r} G \cong f^{*}\left(A \rtimes_{r} G\right)$ for all continuous $f: \Delta^{p} \rightarrow X$. By the assumption on the compact subgroups of $G$ we see that the quotient map $q_{v}: f^{*} A \rightarrow A_{f(v)}$ induces an isomorphism

$$
\mathrm{K}_{*}\left(f^{*} A \rtimes K\right) \stackrel{\cong}{\cong} \mathrm{K}_{*}\left(A_{f(v)} \rtimes K\right)
$$

for all compact subgroups $K$ of $G$. Item (i) then follows from part (i) of Proposition 2.1.

Similarly, (ii) and (iii) follow from parts (ii) and (iii) of Proposition 2.1 together with the fact that the $C_{0}(X)$-algebra $A(X) \rtimes G$ has fibres $A_{x} \rtimes G$. If $G$ is exact, the same argument works for $A(X) \rtimes_{r} G$.

Remark 2.3. (1) If $G$ has no compact subgroups (e.g., $G=\mathbb{R}^{n}, G=\mathbb{Z}^{n}$ or $G=F_{n}$, the free group with $n$ generators), then the requirement that $A(X) \rtimes K$ being a $\mathrm{K}_{*}$-fibration (resp. KK-fibration) in the above proposition reduces to the requirement that $A(X)$ is a $\mathrm{K}_{*}$-fibration (resp. $\mathrm{KK}$-fibration). Therefore, if any of the groups $G=\mathbb{R}^{n}, \mathbb{Z}^{n}, F_{n}$ acts fibrewise on a $\mathrm{K}_{*}$-fibration (resp. KK-fibration) $A(X)$, then $A(X) \rtimes_{(r)} G$ is also a $\mathrm{K}_{*}$-fibration (resp. KK-fibration), since all of these groups are exact and satisfy the strong Baum-Connes conjecture. Of course, there are many other examples of such groups. 
(2) It follows from [3], Proposition 3.1, that if $G$ is exact and has a $\gamma$-element in the sense of Kasparov [17], and if $A(X)$ is a continuous $\mathrm{C}^{*}$-algebra bundle over $X$, then $G$ satisfies $B C$ for $f^{*} A$ for all $f: \Delta^{p} \rightarrow X$ if (and only if) $G$ satisfies $\mathrm{BC}$ for $A_{x}$ for every fibre $A_{x}$ of $A$. (The only if direction follows from taking the constant map $f: \Delta^{p} \rightarrow X ; f(v)=x$ and using the fact that $f^{*} A=C\left(\Delta^{p}, A_{x}\right)$ is $\mathrm{KK}^{G}$-equivalent to $A_{x}$ ).

If we specialize to continuous-trace algebras $A$ with base $X$, we can improve the results. For notation, we let $\mathcal{K}=\mathcal{K}\left(l^{2}(\mathbb{N})\right)$ denote the compact operators on the infinite dimensional separable Hilbert space. Recall that if $A(X)$ is any separable continuous-trace algebra with spectrum $X$, then $A(X) \otimes \mathcal{K}$ is a locally trivial $\mathrm{C}^{*}$ algebra bundle with fibre $\mathcal{K}$. Using this we obtain

Corollary 2.4. Suppose that $G$ is a second countable locally compact group acting fibrewise on a separable continuous-trace $C^{*}$-algebra $A(X)$ with spectrum $X$. Then the following holds:

(i) If $G$ satisfies the strong Baum-Connes conjecture (e.g., if $G$ is a-T-menable), then $A(X) \rtimes G$ is a KK-fibration. If, in addition, $G$ is exact, the same holds for $A \rtimes_{r} G$.

(ii) If $G$ is exact and satisfies $B C$ for $C\left(\Delta^{p}, \mathcal{K}\right)$ for all fibrewise actions on $C\left(\Delta^{p}, \mathcal{K}\right), p \geq 0$, then $A \rtimes_{r} G$ is a $\mathrm{K}_{*}$-fibration.

(iii) If $G$ is exact and has a $\gamma$-element, and $G$ satisfies $B C$ for $\mathcal{K}$, for all actions of $G$ on $\mathcal{K}$, then $A \rtimes_{r} G$ is a $\mathrm{K}_{*}$-fibration.

Notice that by the results of [3] condition (iii) is satisfied for all almost connected groups and for all linear algebraic groups over $\mathbb{Q}_{p}$.

Proof of Corollary 2.4. The corollary follows from Proposition 2.2 and Remark 2.3 if we can show that $\left(f^{*} A \rtimes K\right) \otimes \mathcal{K}$ is a trivial $C\left(\Delta^{p}\right)$-algebra for all continuous maps $f: \Delta^{p} \rightarrow X$, since this implies that $A \rtimes K$ is a KK-fibration.

For this we first note that $\left(f^{*} A \rtimes K\right) \otimes \mathcal{K} \cong\left(f^{*} A \otimes \mathcal{K}\right) \rtimes K$, where $K$ acts trivially on $\mathcal{K}$. Using this we may simply assume that $f^{*} A=C\left(\Delta^{p}, \mathcal{K}\right)$. But it then follows from [9], Proposition 1.5, that any fibrewise action of a compact group $K$ on $C\left(\Delta^{p}, \mathcal{K}\right)=C\left(\Delta^{p}\right) \otimes \mathcal{K}$ is exterior equivalent to a diagonal action id $\otimes \alpha^{v}$, with $\alpha^{v}$ the action on the fibre $\mathcal{K}=C\left(\Delta^{p}, \mathcal{K}\right)_{v}$. Thus $C\left(\Delta^{p}, \mathcal{K}\right) \rtimes K$ is isomorphic to $C\left(\Delta^{p}, \mathcal{K} \rtimes_{\alpha^{v}} K\right)$ as bundle over $\Delta^{p}$.

So far we have only considered $\mathrm{K}_{*^{-}}$or KK-fibrations, but we promised at the beginning of this section that we will provide also examples of $\mathcal{R K K}$-fibrations. Indeed, combining the above results with Corollary 1.6 gives 
Corollary 2.5. Suppose that $A(X)$ is a separable nuclear and locally trivial $C^{*}$ algebra bundle and let $G$ be a second countable amenable group acting fibrewise on $A(X)$. Then the following holds:

(i) If $G$ has no compact subgroups then $A(X) \rtimes G$ is an $\mathcal{R K K}$-fibration.

(ii) If $A(X)$ is a continuous-trace algebra with spectrum $X$, then $A(X) \rtimes G$ is an RKK-fibration.

Proof. Since $G$ is amenable, it satisfies the strong Baum-Connes conjecture by [14]. Moreover, a crossed product of a continuous $\mathrm{C}^{*}$-algebra bundle by a fibrewise group action of an amenable group $G$ is again a continuous $C^{*}$-algebra bundle by [23]. Since nuclearity is also preserved under taking crossed products by amenable groups, it follows that $A(X) \rtimes G$ is a nuclear separable and continuous $\mathrm{C}^{*}$-algebra bundle. Hence Corollary 1.6 implies that $A(X) \rtimes G$ is an $\mathcal{R K K}$-fibration if and only if it is a KK-fibration. Thus the result follows from Remark 2.3 and Corollary 2.4.

Of course, as an example of the above corollary we get a new proof of the fact that the noncommutative principal torus bundles of [10] are $\mathcal{R K K}$-fibrations, since, by definition, they are crossed products of the form $C_{0}(X, \mathcal{K}) \rtimes \mathbb{Z}^{n}$ by $C_{0}(X)$-linear actions of $\mathbb{Z}^{n}$ on $C_{0}(X, \mathcal{K})$.

\section{The group bundle corresponding to an $h$-fibration}

Let $X$ be a locally compact space. By an (abelian) group bundle $\mathscr{E}:=\left\{G_{x} \mid x \in X\right\}$ we understand a functor from the homotopy groupoid of $X$ to the category of (abelian) groups. It is given by a family of groups $G_{x}, x \in X$, together with group isomorphisms $c_{\gamma}: G_{x} \rightarrow G_{y}$ for each continuous path $\gamma:[0,1] \rightarrow X$ which starts at $x$ and ends at $y$, such that the following additional requirements are satisfied:

(i) If $\gamma$ and $\gamma^{\prime}$ are homotopic paths from $x$ to $y$, then $c_{\gamma}=c_{\gamma^{\prime}}$.

(ii) If $\gamma_{1}:[0,1] \rightarrow X$ and $\gamma_{2}:[0,1] \rightarrow X$ are paths from $x$ to $y$ and from $y$ to $z$, respectively, then

$$
c_{\gamma_{1} \circ \gamma_{2}}=c_{\gamma_{1}} \circ c_{\gamma_{2}}
$$

where $\gamma_{1} \circ \gamma_{2}:[0,1] \rightarrow X$ is the usual composition of paths.

It follows from the above requirements that if $X$ is path connected, then all groups $G_{x}$ are isomorphic and that we get a canonical action of the fundamental group $\pi_{1}(X)$ on each fibre $G_{x}$.

A morphism between two group bundles $\mathscr{G}=\left\{G_{x} \mid x \in X\right\}$ and $\mathscr{G}^{\prime}=\left\{G_{x}^{\prime} \mid x \in\right.$ $X\}$ is a family of group homomorphisms $\phi_{x}: G_{x} \rightarrow G_{x}^{\prime}$ which commutes with the maps $c_{\gamma}$. The trivial group bundle is the bundle with every $G_{x}$ equal to a fixed group $G$ and all maps $c_{\gamma}$ being the identity. We then write $X \times G$ for this bundle. If $X$ is 
path connected, then a given group bundle $\mathcal{E}$ on $X$ can be trivialized if and only if the action of $\pi_{1}(X)$ on the fibres $G_{x}$ are trivial. In that case every path $\gamma$ from base points $x$ to $y$ induces the same morphism $c_{x, y}: G_{x} \rightarrow G_{y}$ and if we choose a fixed base point $x_{0}$, the family of maps $\left\{c_{x, x_{0}} \mid x \in X\right\}$ is a group bundle isomorphism between the trivial group bundle $X \times G_{x_{0}}$ and the given bundle $\mathcal{E}=\left\{G_{x} \mid x \in X\right\}$. It is now easy to check that every $h_{*}$-fibration $A(X)$ gives rise to a group bundle $\mathscr{H}_{*}:=\left\{h_{*}\left(A_{x}\right) \mid x \in X\right\}$ :

Proposition 3.1. Suppose that $A(X)$ is an $h_{*}$-fibration. For any path $\gamma:[0,1] \rightarrow X$ with starting point $x$ and endpoint $y$ let $c_{\gamma}: h_{*}\left(A_{x}\right) \rightarrow h_{*}\left(A_{y}\right)$ denote the composition

$$
h_{*}\left(A_{y}\right) \stackrel{\mathrm{ev}_{1, *}^{-1}}{\cong} h_{*}\left(\gamma^{*} A\right) \stackrel{\mathrm{ev}_{0, *}}{\cong} h_{*}\left(A_{x}\right)
$$

Then $\mathscr{H}_{*}(A):=\left\{h_{*}\left(A_{x}\right) \mid x \in X\right\}$ together with the above defined maps $c_{\gamma}$ is a group bundle over $X$. A similar result holds for a cohomology theory $h^{*}$ if $A(X)$ is an $h^{*}$-fibration (with arrows in (3.1) reversed).

Proof. It is clear that constant paths induce the identity maps and that $c_{\gamma \circ \gamma^{\prime}}=c_{\gamma} \circ c_{\gamma^{\prime}}$, where $\gamma \circ \gamma^{\prime}$ denotes composition of paths. Moreover, if $\Gamma:[0,1] \times[0,1] \rightarrow X$ is a homotopy between the paths $\gamma_{0}$ and $\gamma_{1}$ with equal starting and endpoints, then $c_{\gamma_{0}}$ and $c_{\gamma_{1}}$ both coincide with the composition $\mathrm{ev}_{(0,0), *} \circ \mathrm{ev}_{(1,1), *}^{-1}$, where $\mathrm{ev}_{(0,0)}$ and $\mathrm{ev}_{(1,1)}$ denote evaluations of $\Gamma^{*} A$ at the respective corners of $[0,1]^{2}$. Hence we see that $c_{\gamma}$ only depends on the homotopy class of $\gamma$.

Definition 3.2. Suppose that $h$ is a (co)homology theory on a good category $\ell$ of $\mathrm{C}^{*}$-algebras and let $A(X)$ be an $h$-fibration. Then $\mathscr{H}_{*}:=\left\{h_{*}\left(A_{x}\right) \mid x \in X\right\}$ (resp. $\mathscr{H}^{*}(A):=\left\{h^{*}\left(A_{x}\right) \mid x \in X\right\}$ if $h$ is a cohomolgy theory) together with the maps $c_{\gamma}: h_{*}\left(A_{y}\right) \rightarrow h_{*}\left(A_{x}\right)$ is called the $h_{*}$-group bundle associated to $A(X)$.

Remark 3.3. If $A(X)$ is a KK-fibration, then it is in particular a $\mathrm{K}_{*^{-}}$and a $\mathrm{K}^{*}$ fibration, where $\mathrm{K}_{*}$ and $\mathrm{K}^{*}$ denote ordinary $\mathrm{K}$-theory and $\mathrm{K}$-homology. We shall denote the resulting group bundles by $\mathcal{K}_{*}(A)$ and $\mathcal{K}^{*}(A)$, respectively.

\section{The Leray-Serre spectral sequence}

In this section we want to prove an analogue of the classical Leray-Serre spectral sequence for topological Serre fibrations. From the last remark of the previous section we know that if $A(X)$ is a $h$-fibration for a (co-)homology theory $h$, then we get the group bundle $\mathcal{H}_{*}(A)$ over $X$. It is well known in topology that one can use such bundles as coefficients for singular or simplicial (co-)homology on $X$. It is our aim to show that every $h$-fibration over a finite dimensional simplicial complex $X$ 
admits a spectral sequence with $E_{2}$-terms isomorphic to the (co-)homology of $X$ with coefficient in $\mathscr{H}_{*}\left(\right.$ resp. $\left.\mathscr{H}^{*}\right)$.

Assume that $X$ is a locally compact CW-complex and that $A$ is any $C_{0}(X)$-algebra. For $p \geq 0$ let $X_{p}$ denote the $p$-skeleton of $X$ and we set $X_{p}=\emptyset$ for a negative integer $p<0$. We will always assume that $X$ is finite dimensional so that there exists a smallest integer $d$ (the dimension of $X$ ) such that $X_{p}=X$ for all $p \geq d$. For all $p$ we write $A_{p}:=\left.A\right|_{X_{p}}$ and $A_{p, p-1}:=\left.A\right|_{X_{p} \backslash X_{p-1}}$, where we use $\left.A\right|_{\emptyset}:=\{0\}$. We then obtain short exact sequences

$$
0 \rightarrow A_{p, p-1} \rightarrow A_{p} \rightarrow A_{p-1} \rightarrow 0 .
$$

If $h_{*}$ is any homology theory on a good subcategory $\mathcal{C}_{\text {of }} \mathcal{C}_{\text {all }}$ such that all algebras $A_{p}$ and $A_{p, p-1}$ are in $\mathcal{C}$, naturality of the long exact sequences

$$
\cdots \stackrel{\partial_{n+1}}{\longrightarrow} h_{n}(J) \stackrel{i_{*}}{\longrightarrow} h_{n}(A) \stackrel{q_{*}}{\longrightarrow} h_{n}(B) \stackrel{\partial_{n}}{\longrightarrow} h_{n-1}(J) \stackrel{i_{*}}{\longrightarrow} \cdots
$$

gives the following commutative diagram:

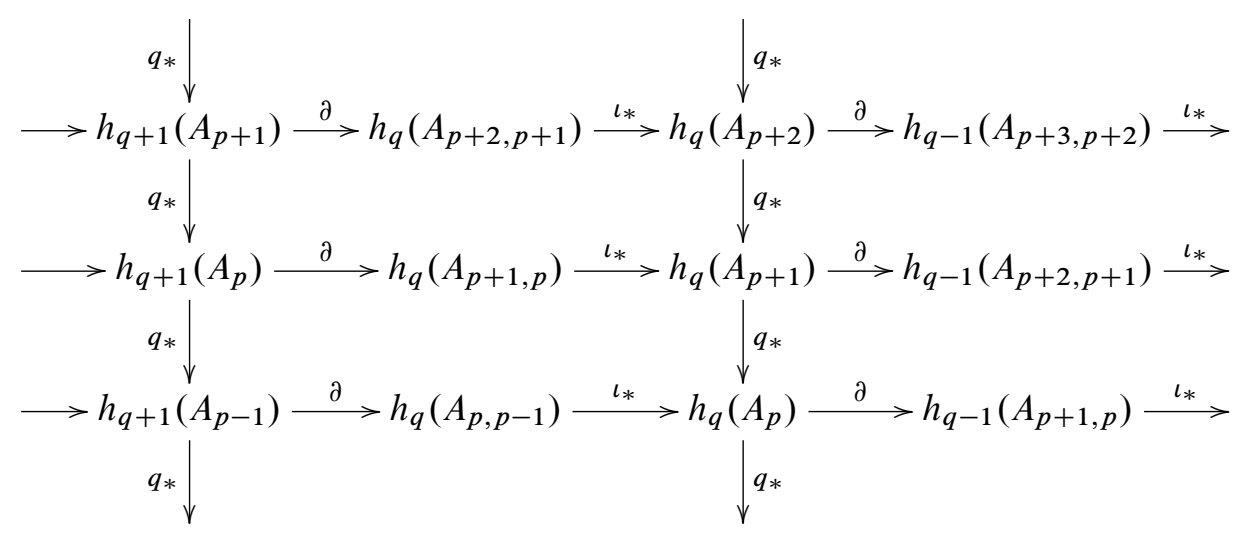

Here the vertical arrows are induced by the quotient maps $q: A_{p} \rightarrow A_{p-1}$, the maps $\iota^{*}: h_{q}\left(A_{p, p-1}\right) \rightarrow h_{q}\left(A_{p}\right)$ are induced by the inclusions $\iota: A_{p, p-1} \rightarrow A_{p}$ and the maps $\partial: h_{q+1}\left(A_{p-1}\right) \rightarrow h_{q}\left(A_{p, p-1}\right)$ denote the boundary maps in the long exact sequence (4.1). Hence, the upper staircase of this diagram forms the sequence (4.1). Now writing $H^{p, q}:=h_{q}\left(A_{p}\right), E_{1}^{p, q}:=h_{q}\left(A_{p, p-1}\right), \mathscr{H}:=\bigoplus_{p, q} H^{p, q}$ and $\mathcal{E}:=\bigoplus E_{1}^{p, q}$ we obtain an exact couple

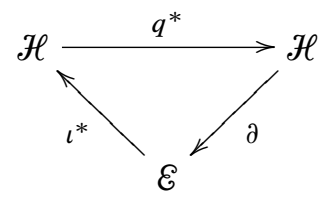


from which we get by the general procedure (explained, for instance, in [19]) a spectral sequence $\left\{E_{r}^{p, q}, d_{r}\right\}$ with $E_{\infty}$-terms $E_{\infty}^{p, q}=F_{p}^{q} / F_{p+1}^{q}$ with $F_{p}^{q}:=\operatorname{ker}\left(h_{q}(A) \rightarrow\right.$ $\left.h_{q}\left(A_{p}\right)\right)$. Since $F_{p}^{q}=h_{q}(A)$ for $p<0$ and $F_{p}^{q}=\{0\}$ for $p \geq d$, the dimension of $X$, it follows that the spectral sequence converges to $h_{q}(A)$. This means that we obtain a filtration

$$
\{0\}=F_{d}^{q} \subseteq F_{d-1}^{q} \subseteq \cdots \subseteq F_{-1}^{q}=h_{q}(A)
$$

of subgroups $F_{p}^{q}$ of $h_{q}(A)$ such that the sub-quotients can be computed (at least in principle) by our spectral sequence.

Similarly, if we start with a cohomology theory $h^{*}$ on $\ell$, we consider the diagram

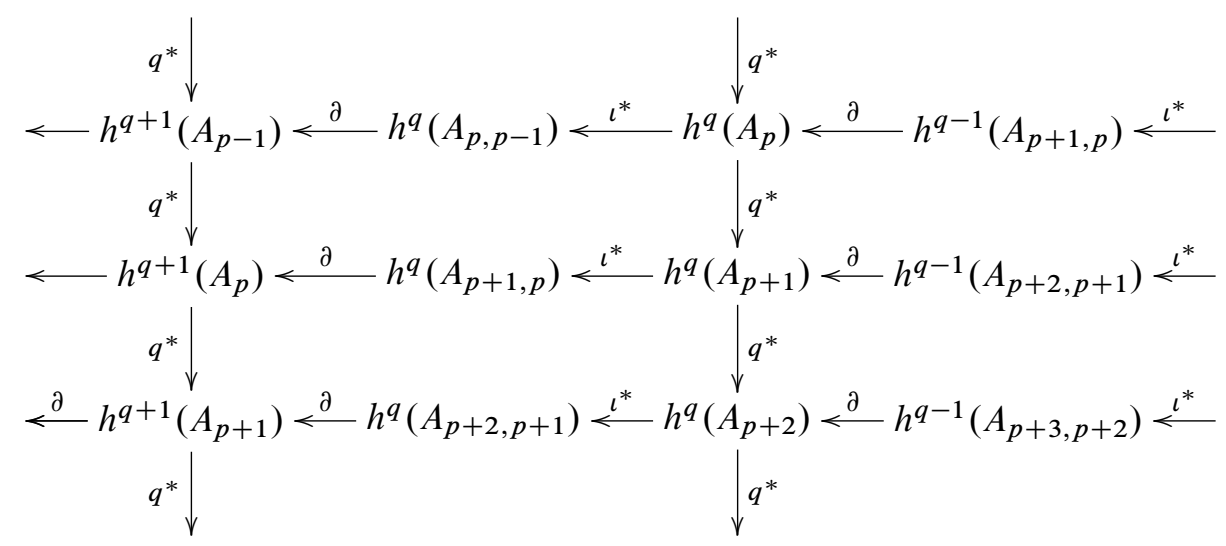

which provides a spectral sequence $\left\{E_{p, q}^{r}, d^{r}\right\}$ with $E^{\infty}$-terms $E_{p, q}^{\infty}:=F_{q}^{p} / F_{q}^{p-1}$ where $F_{q}^{p}:=\operatorname{im}\left(h^{q}\left(A_{p}\right) \rightarrow h^{q}(A)\right)$. Again, since $X$ is finite dimensional, the spectral sequence converges to $h^{q}(A)$. Hence, at this stage we arrive at

Proposition 4.1. Suppose that $X$ is a finite dimensional $C W$-complex and let $A(X)$

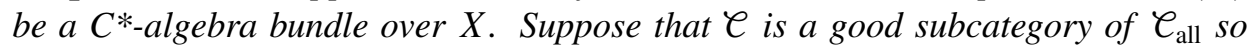
that $A_{p}:=A\left(X_{p}\right) \in \mathcal{C}$ for every $p$-skeleton of $X$. Then if $h_{*}$ is a homology theory (resp. $h^{*}$ is a cohomology theory) on $X$, there exists a spectral sequence $\left\{E_{r}^{p, q}, d_{r}\right\}$ (resp. $\left.\left\{E_{p, q}^{r}, d^{r}\right\}\right)$ which converges to $h_{*}(A)$ (resp. $\left.h^{*}(A)\right)$ as described above.

Remark 4.2. Let us denote by $\left\{U_{i}^{p} \mid i \in I_{p}\right\}$ the open $p$-cells of $X$. We then have

$$
A_{p, p-1}=\bigoplus_{i \in I_{p}} A\left(U_{i}^{p}\right)
$$

If $X$ is a finite simplicial complex this sum is finite and it follows from additivity of $h_{*}\left(\right.$ resp. $\left.h^{*}\right)$ that

$$
E_{1}^{p, q}=\bigoplus_{i \in I_{p}} h_{q}\left(A\left(U_{i}^{p}\right)\right) \quad\left(\text { resp. } E_{p, q}^{1}=\bigoplus_{i \in I_{p}} h^{q}\left(A\left(U_{i}^{p}\right)\right)\right) .
$$


Of course, if $h_{*}\left(\right.$ resp. $\left.h^{*}\right)$ is $\sigma$-additive or $\sigma$-multiplicative we get similar infinite direct sum or product decompositions in case where $X$ is $\sigma$-finite (i.e., $X$ has countably many cells). In any case we shall assume that $X$ is locally finite. The $d_{1}$-differential is then determined by the maps

$$
d_{1, q}^{(p, i),(p+1, j)}: h_{q}\left(A\left(U_{i}^{p}\right)\right) \rightarrow h_{q-1}\left(A\left(U_{j}^{p+1}\right)\right)
$$

given by the composition

$$
\begin{aligned}
h_{q}\left(A\left(U_{i}^{p}\right)\right) & \stackrel{I_{q}^{p, i}}{\longrightarrow} h_{q}\left(A_{p, p-1}\right) \stackrel{i_{*}}{\rightarrow} h_{q}\left(A_{p}\right) \\
& \stackrel{\partial}{\rightarrow} h_{q-1}\left(A_{p+1, p}\right) \stackrel{Q_{q-1}^{p+1, j}}{\longrightarrow} h_{q-1}\left(A\left(U_{j}^{p+1}\right)\right),
\end{aligned}
$$

where $I^{p, i}: A\left(U_{i}^{p}\right) \rightarrow A_{p, p-1}$ denotes the inclusion and $Q^{p, i}: A_{p, p-1} \rightarrow A\left(U_{i}^{p}\right)$ denotes the quotient map. Similarly, for a cohomology theory $h^{*}$ we get maps

$$
d_{(p, i),(p-1, j)}^{1, q}: h^{q}\left(A\left(U_{i}^{p}\right)\right) \rightarrow h^{q+1}\left(A\left(U_{j}^{p-1}\right)\right),
$$

which are given by the compositions

$$
\begin{aligned}
h^{q}\left(A\left(U_{i}^{p}\right)\right) & \stackrel{Q_{p, i}^{q}}{\longrightarrow} h^{q}\left(A_{p, p-1}\right) \stackrel{\partial}{\rightarrow} h^{q+1}\left(A_{p-1}\right) \\
& \stackrel{i^{*}}{\longrightarrow} h^{q+1}\left(A_{p-1, p-2}\right) \stackrel{I_{p-1, j}^{q+1}}{\longrightarrow} h^{q+1}\left(A\left(U_{j}^{p-1}\right)\right)
\end{aligned}
$$

with similar meanings for $Q_{p, i}$ and $I_{p, i}$. It follows then that $E_{2}^{p, q}$ (resp. $E_{p, q}^{2}$ ) is the cohomology (resp. homology) of the complex build out of the above given data.

We now want to study the groups $h_{*}\left(A\left(U_{i}^{p}\right)\right)$ and the maps $d_{1, q}^{(p, i),(p+1, j)}$ more closely in case where $A(X)$ is an $h_{*}$-fibration and $X$ is a (finite) simplicial complex. In particular, we want to give a better computation of the $E_{2}$-terms. We shall restrict to the case of a homology theory on a good category $\mathcal{C}$ of $\mathrm{C}^{*}$-algebras throughout, noting that similar arguments work for a cohomology theory as well.

We start with introducing some notation: As before, we let

$$
\Delta^{p}:=<v_{0}, \ldots, v_{p}>
$$

denote the oriented closed $n$-simplex with vertices $v_{0}, \ldots, v_{p}$, we let $\stackrel{\circ}{\Delta}^{p}$ denote its interior and we let $\partial \Delta^{p}$ denote its boundary. If $0 \leq k \leq p$ we shall consider $\Delta^{k}=<v_{0}, \ldots, v_{k}>$ as a subset of $\Delta^{p}$ and for $i \in\{0, \ldots, n\}$ we write $\Delta_{i}^{p-1}:=$ $<v_{0}, \ldots, v_{i-1}, v_{i+1}, \ldots, v_{p}>$ for the oriented $i$ th face of $\Delta^{p}$. If $X$ is our given simplicial complex we write $\left\{\Delta^{p}(j) \mid j \in I_{p}\right\}$ for the set of closed $p$-simplexes in $X$, and we let

$$
\sigma^{p}(j): \Delta^{p} \rightarrow \Delta^{p}(j) \subseteq X
$$


denote an explicit affine homeomorphism between the standard simplex $\Delta^{p}$ and $\Delta^{p}(j)$.

To study the differential $d: E_{p, q}^{1} \rightarrow E_{p+1, q-1}^{2}$ in the above remark, we first need to study the simple case where $X=\Delta^{p}$ itself. Recall that if $A=A(X)$ is an $h_{*}$-fibration with $X$ simply connected, then for each $x, y \in X$ there are unique isomorphisms $\Phi_{x, y}: h_{*}\left(A_{x}\right) \rightarrow h_{*}\left(A_{y}\right)$, which, for any chosen path $\gamma:[0,1] \rightarrow X$ with $\gamma(0)=y, \gamma(1)=x$, satisfy the equations

$$
\Phi_{x, y} \circ \mathrm{ev}_{1, *}=\mathrm{ev}_{0, *}: h_{*}\left(\gamma^{*} A[0,1]\right) \rightarrow h_{*}\left(A_{y}\right) .
$$

Lemma 4.3. Suppose that $A=A\left(\Delta^{P}\right)$ is an $h_{*}$-fibration (resp. $h^{*}$-fibration) with $p \geq 1$. Then for every $x \in \Delta^{p}$ evaluation at $x$ induces an isomorphism

$$
\mathrm{ev}_{x, *}: h_{*}\left(A\left(\Delta^{p}\right)\right) \rightarrow h_{*}\left(A_{x}\right)
$$

Moreover, if $y$ is any other point in $\Delta^{p}$, then $\mathrm{ev}_{y, *}=\Phi_{x, y} \circ \mathrm{ev}_{x, *}$ (and similar statements for $h^{*}$-fibrations).

Proof. The first statement holds by definition of an $h_{*}$-fibration. So we only have to check that $\mathrm{ev}_{y, *}=\Phi_{x, y} \circ \mathrm{ev}_{x, *}$ for any pair $x, y \in \Delta^{p}$. Let $\gamma:[0,1] \rightarrow \Delta^{p}$ denote any path connecting $y=\gamma(0)$ with $x=\gamma(1)$. Since $\gamma$ is a proper map, [10], Lemma 1.3, provides a ${ }^{*}$-homomorphism $\Phi_{\gamma}: A\left(\Delta^{p}\right) \rightarrow \gamma^{*} A([0,1])$, and it is clear from the construction of $\Phi_{\gamma}$ that $\mathrm{ev}_{x}=\mathrm{ev}_{1} \circ \Phi_{\gamma}$ and $\mathrm{ev}_{y}=\mathrm{ev}_{0} \circ \Phi_{\gamma}$. The result now follows from the definition of $\Phi_{x, y}$.

Lemma 4.4. Let $1 \leq p$ and suppose that the $C\left(\Delta^{p}\right)$-algebra $A$ is an $h_{*}$-fibration. Let $W \subseteq \Delta^{p}$ be any set which is obtained from $\Delta^{p}$ by removing a union of $k$ faces of dimension $p-1$ from $\Delta^{p}$ with $1 \leq k \leq p$. Then $h_{*}(A(W))=0$.

Proof. The proof is by induction on $p$ and $k$. If $p=1$ then $k=1$ and $W$ is homeomorphic to $[0,1)$. Since evaluation $A([0,1]) \rightarrow A_{1}$ induces an isomorphism of $h_{*}$-groups, it follows from the long exact sequence corresponding to $0 \rightarrow A([0,1)) \rightarrow$ $A([0,1]) \rightarrow A_{1} \rightarrow 0$ that $h_{*}(A(W))=0$.

Suppose now that $p>1$. If $\Delta_{i}^{p-1}$ is any closed face of $\Delta^{p}$, then it follows from the properties of $h_{*}$-fibrations that the quotient map $q_{i}: A\left(\Delta^{p}\right) \rightarrow A\left(\Delta_{i}^{p-1}\right)$ induces an isomorphism $h_{n}\left(A\left(\Delta^{p}\right)\right) \stackrel{q_{i, *}}{\longrightarrow} h_{n}\left(A\left(\Delta_{i}^{p-1}\right)\right)$, since composition with evaluation at any vertex $v$ of $\Delta_{i}^{p-1}$ induces isomorphisms $h_{n}\left(A\left(\Delta^{p}\right)\right) \cong h_{n}\left(A\left(\Delta_{i}^{p-1}\right)\right) \cong h_{n}\left(A_{v}\right)$. Hence, if $W=\Delta^{p} \backslash \Delta_{i}^{p-1}$, the long exact sequence of $h_{*}$-groups corresponding to the short exact sequence $0 \rightarrow A(W) \rightarrow A\left(\Delta^{p}\right) \rightarrow A\left(\Delta_{i}^{p-1}\right) \rightarrow 0$ implies that $h_{*}(A(W))=0$.

Suppose now that $k>1$ and let $F:=\Delta^{p} \backslash W$. Then we can write $F$ as a union $F^{\prime} \cup \Delta_{i}^{p-1}$, where $F^{\prime}$ is a union of $k-1$ faces. By the induction assumption 
we know that $h_{*}\left(A\left(W^{\prime}\right)\right)=0$ for $W^{\prime}:=\Delta^{p} \backslash F^{\prime}$. Moreover, $W^{\prime} \backslash W$ is equal to $W^{\prime \prime}:=\Delta_{i}^{p-1} \backslash F^{\prime \prime}$, where $F^{\prime \prime}$ is some union of $l(p-2)$-dimensional faces with $1 \leq l \leq p-1$. Hence, by induction assumption, we have $h_{*}\left(A\left(W^{\prime \prime}\right)\right)=0$ and then all terms in the exact sequence $h_{q}\left(A\left(W^{\prime}\right)\right) \rightarrow h_{q}(A(W)) \rightarrow h_{q}\left(A\left(W^{\prime \prime}\right)\right)$ must be zero.

Definition and Remark 4.5. For the $p$-simplex $\Delta^{p}=<v_{0}, \ldots, v_{p}>$ we denote $W_{i}:=\stackrel{\circ}{\Delta}^{p} \cup \stackrel{\circ}{\Delta}_{i}^{p-1}$. If the $C\left(\Delta^{p}\right)$-algebra $A$ is an $h_{*}$-fibration, then it follows from the above lemma that $h_{*}\left(A\left(W_{i}\right)\right)=0$, which then implies that the boundary map $\partial_{i}: h_{q}\left(A\left(\stackrel{\circ}{\Delta}_{i}^{p-1}\right)\right) \rightarrow h_{q-1}\left(A\left(\stackrel{\circ}{\Delta}^{p}\right)\right)$ corresponding to the short exact sequence $0 \rightarrow A\left(\stackrel{\circ}{\Delta}^{p}\right) \rightarrow A\left(W_{i}\right) \rightarrow A\left(\stackrel{\circ}{\Delta}_{i}^{p-1}\right) \rightarrow 0$ is an isomorphism for all $q$.

In particular, there is a chain of isomorphisms

$$
h_{q}\left(A\left(\Delta^{p}\right)\right) \stackrel{\mathrm{ev}_{v_{0}, *}}{\longrightarrow} h_{q}\left(A_{v_{0}}\right) \stackrel{\partial_{1}}{\longrightarrow} h_{q-1}\left(A\left(\stackrel{\circ}{\Delta}^{1}\right)\right) \stackrel{\partial_{2}}{\longrightarrow} \ldots \stackrel{\partial_{p}}{\longrightarrow} h_{q-p}\left(A\left(\stackrel{\circ}{\Delta}^{p}\right)\right),
$$

which we shall call the canonical oriented isomorphism

$$
\Phi_{q}^{p}: h_{q}\left(A\left(\Delta^{p}\right)\right) \stackrel{\cong}{\longrightarrow} h_{q-p}\left(A\left(\stackrel{\circ}{\Delta}^{p}\right)\right) .
$$

It is important for us to understand how the canonical isomorphisms depends on the orientation of the simplex $\Delta^{p}$. We start this investigation with two basic observations. The first considers the case $\Delta^{1}=[0,1]$ :

Lemma 4.6. Suppose that $A([0,1])$ is a $C^{*}$-algebra bundle which is an $h_{*}$-fibration. Let

$$
\partial_{0}: h_{q}\left(A_{0}\right) \rightarrow h_{q-1}(A(0,1)) \text { and } \partial_{1}: h_{q}\left(A_{1}\right) \rightarrow h_{q-1}(A(0,1))
$$

denote the isomorphisms given by the connecting maps in the long exact sequences related to evaluation of $A([0,1))$ and $A((0,1])$ at 0 and 1 , respectively. Then $\partial_{0}=-\partial_{1} \circ \Phi_{0,1}$.

Proof. Consider the long exact sequence

$$
\cdots \rightarrow h_{q}(A(0,1)) \rightarrow h_{q}(A[0,1]) \rightarrow h_{q}\left(A_{0} \oplus A_{1}\right) \stackrel{\partial}{\rightarrow} h_{q-1}(A(0,1)) \rightarrow \cdots
$$

corresponding to

$$
0 \rightarrow A((0,1)) \rightarrow A([0,1]) \rightarrow A_{0} \oplus A_{1} \rightarrow 0 .
$$

The connecting map in this sequence equals $\partial_{0}+\partial_{1}$, which follows from naturality 
of the long exact sequence together with the diagram

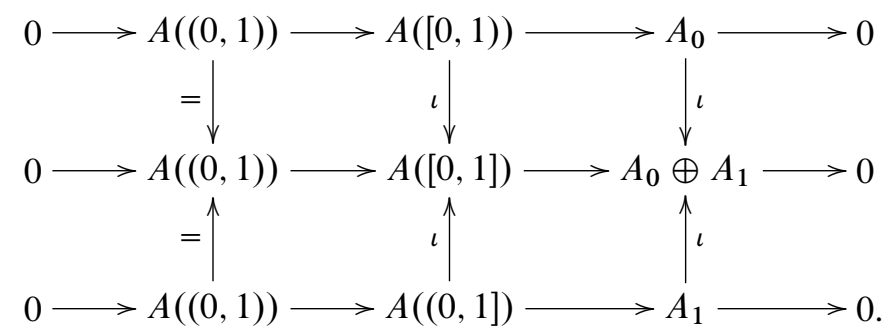

Exactness then gives

$$
0=\partial_{0} \circ \mathrm{ev}_{0, *}+\partial_{1} \circ \mathrm{ev}_{1, *},
$$

where $\mathrm{ev}_{i, *}: h_{q}(A([0,1])) \rightarrow h_{q}\left(A_{i}\right), i=0,1$, denotes the evaluation isomorphism. Thus we get $\partial_{0} \circ \mathrm{ev}_{0, *}=-\partial_{1} \circ \mathrm{ev}_{1, *}$ and composing both sides with $\mathrm{ev}_{0, *}^{-1}$ on the right gives the lemma.

In the next lemma we compare the isomorphisms

$$
h_{q}\left(A\left(\stackrel{\circ}{\Delta}_{p-1}^{p-1}\right)\right) \cong h_{q-1}\left(A\left(\stackrel{\circ}{\Delta}^{p}\right)\right)
$$

and

$$
h_{q}\left(A\left(\stackrel{\circ}{\Delta}_{p}^{p-1}\right)\right) \cong h_{q-1}\left(A\left(\stackrel{\circ}{\Delta}^{p}\right)\right),
$$

as defined in 4.5 for the $p$-th and the $(p-1)$-st face of $\Delta^{p}$ :

Lemma 4.7. Suppose that $p>1$. Then the compositions

$$
h_{r}\left(A\left(\stackrel{\circ}{\Delta}^{p-2}\right)\right) \cong h_{r-1}\left(A\left(\stackrel{\circ}{\Delta}_{p}^{p-1}\right)\right) \cong h_{r-2}\left(A\left(\stackrel{\circ}{\Delta}^{p}\right)\right)
$$

and

$$
h_{r}\left(A\left(\stackrel{\circ}{\Delta}^{p-2}\right)\right) \cong h_{r-1}\left(A\left(\stackrel{\circ}{\Delta}_{p-1}^{p-1}\right)\right) \cong h_{r-2}\left(A\left(\stackrel{\circ}{\Delta}^{p}\right)\right)
$$

differ by the factor -1 .

Proof. Let

$$
\begin{aligned}
W & =\stackrel{\circ}{\Delta}^{p-2} \cup \stackrel{\circ}{\Delta}_{p}^{p-1} \cup \stackrel{\circ}{\Delta}_{p-1}^{p-1} \cup \stackrel{\circ}{\Delta}^{p}, & W_{0} & =\stackrel{\circ}{\Delta}^{p-2} \cup \stackrel{\circ}{\Delta}_{p}^{p-1}, \\
W_{1} & =\stackrel{\circ}{\Delta}^{p-2} \cup \stackrel{\circ}{\Delta}_{p-1}^{p-1}, & W_{0,1} & =\stackrel{\circ}{\Delta}_{p}^{p-1} \cup \stackrel{\circ}{\Delta}_{p-1}^{p-1} \cup \stackrel{\circ}{\Delta}^{p-2} .
\end{aligned}
$$

Then it follows from Lemma 4.4 that all groups

$$
h_{*}(A(W))=h_{*}\left(A\left(W_{1}\right)\right)=h_{*}\left(A\left(W_{0}\right)\right)=h_{*}\left(\stackrel{\circ}{\Delta}^{p} \cup \stackrel{\circ}{\Delta}_{p}^{p-1}\right)=h_{*}\left(\stackrel{\circ}{\Delta}^{p} \cup \stackrel{\circ}{\Delta}_{p-1}^{p-1}\right)
$$


vanish. Since $h_{*}(A(W))=0$, the boundary map in the long exact sequence for

$$
0 \rightarrow A\left(\stackrel{\circ}{\Delta}^{p}\right) \rightarrow A(W) \rightarrow A\left(W_{0,1}\right) \rightarrow 0
$$

induces an isomorphism

$$
\partial_{0,1}: h_{l}\left(A\left(W_{0,1}\right)\right) \rightarrow h_{l-1}\left(A\left(\stackrel{\circ}{\Delta}^{p}\right)\right) .
$$

Naturality of the long exact sequences and the diagram

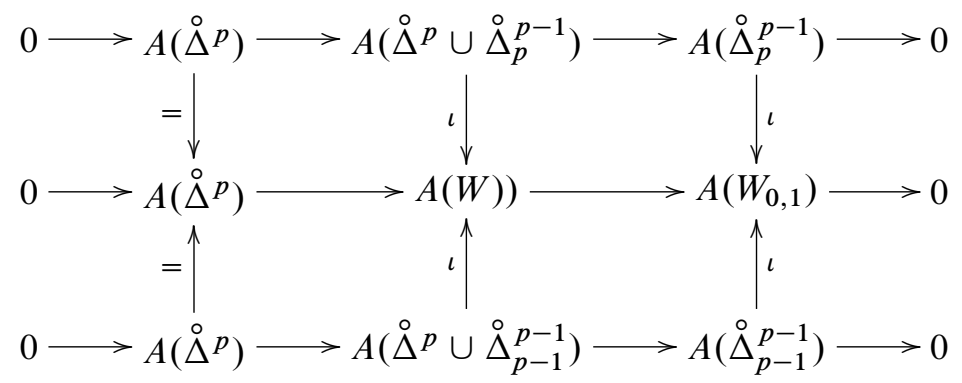

together with the Five-Lemma shows that the inclusions of $\stackrel{\triangle}{\Delta}_{p}^{p-1}$ and $\stackrel{\circ}{\Delta}_{p-1}^{p-1}$ into $W_{0,1}$ induce isomorphisms

$$
\iota_{p}: h_{l}\left(A\left(\stackrel{\circ}{\triangle}_{p}^{p-1}\right)\right) \stackrel{\cong}{\rightrightarrows} h_{l}\left(A\left(W_{0,1}\right)\right)
$$

and

$$
\iota_{p-1}: h_{l}\left(A\left(\stackrel{\circ}{\Delta}_{p-1}^{p-1}\right)\right) \stackrel{\cong}{\cong} h_{l}\left(A\left(W_{0,1}\right)\right)
$$

such that

$$
\partial_{0,1} \circ \iota_{p}=\partial_{p} \quad \text { and } \quad \partial_{0,1} \circ \iota_{p-1}=\partial_{p-1},
$$

where, as before, $\partial_{i}: h_{l}\left(A\left(\stackrel{\circ}{\Delta}_{i}^{p}\right)\right) \rightarrow h_{l-1}\left(A\left(\stackrel{\circ}{\Delta}^{p}\right)\right)$ denotes the isomorphism for the $i$ th face of $\Delta^{p}$. We now look at the diagram

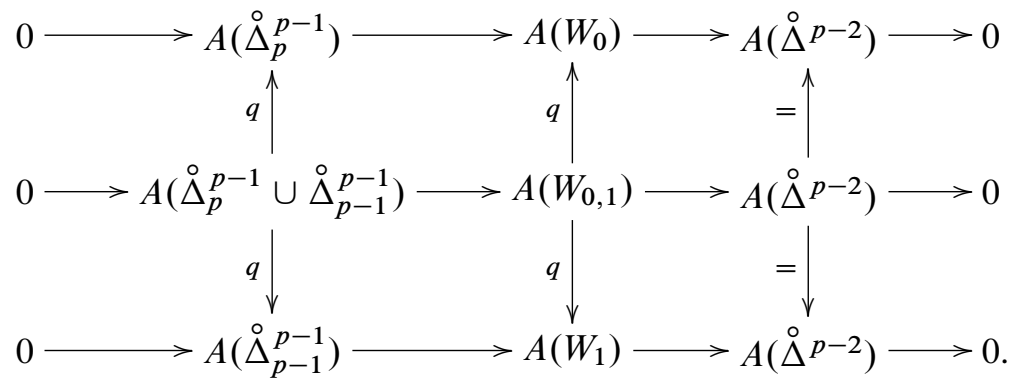

It implies that the composition of the boundary map

$$
h_{r}\left(A\left(\stackrel{\circ}{\Delta}^{p-2}\right)\right) \rightarrow h_{r-1}\left(A\left(\stackrel{\circ}{\Delta}_{p}^{p-1} \cup \stackrel{\circ}{\Delta}_{p-1}^{p-1}\right)\right)
$$


followed by the projections onto $h_{r-1}\left(A\left(\stackrel{\circ}{\Delta}_{p}^{p-1}\right)\right)$ and $h_{r-1}\left(A\left(\stackrel{\circ}{\Delta}_{p-1}^{p-1}\right)\right)$, respectively, coincide with the boundary maps

$$
\partial_{p}^{p-2}: h_{r}\left(A\left(\stackrel{\circ}{\Delta}^{p-2}\right)\right) \cong h_{r-1}\left(A\left(\stackrel{\circ}{\Delta}_{p}^{p-1}\right)\right)
$$

and

$$
\partial_{p-1}^{p-2}: h_{r}\left(A\left(\stackrel{\circ}{\Delta}^{p-2}\right)\right) \cong h_{r-1}\left(A\left(\stackrel{\circ}{\Delta}_{p-1}^{p-1}\right)\right),
$$

respectively. On the other hand, it is clear that the isomorphisms

$$
\iota_{p}: h_{r-1}\left(A\left(\stackrel{\circ}{\Delta}_{p}^{p-1}\right)\right) \cong h_{r-1}\left(A\left(W_{0,1}\right)\right)
$$

and

$$
\iota_{p-1}: h_{r-1}\left(A\left(\stackrel{\circ}{\Delta}_{p-1}^{p-1}\right)\right) \cong h_{r-1}\left(A\left(W_{0,1}\right)\right)
$$

factorise via the inclusions of $h_{r-1}\left(A\left(\stackrel{\circ}{\Delta}_{p}^{p-1}\right)\right)$ and $h_{r-1}\left(A\left(\stackrel{\circ}{\Delta}_{p-1}^{p-1}\right)\right)$ as direct summands of $h_{r-1}\left(A\left(\stackrel{\circ}{\Delta}_{p}^{p-1} \cup \stackrel{\circ}{\Delta}_{p-1}^{p-1}\right)\right)$, and that these inclusions invert the projections on the summands which are induced from the quotient maps in the first vertical row of diagram (4.3). This implies exactness of the sequence

$$
\begin{aligned}
& h_{r}\left(A\left(\stackrel{\circ}{\Delta}^{p-2}\right)\right) \stackrel{\partial_{p}^{p-2} \oplus \partial_{p-1}^{p-2}}{\longrightarrow} h_{r-1}\left(A\left(\stackrel{\circ}{\Delta}_{p}^{p-1}\right)\right) \oplus h_{r-1}\left(A\left(\stackrel{\circ}{\Delta}_{p-1}^{p-1}\right)\right) \\
& \stackrel{\iota_{p}+\iota_{p-1}}{\longrightarrow} h_{r-1}\left(A\left(W_{0,1}\right)\right) .
\end{aligned}
$$

Combining this with eq. (4.2) gives

$$
0=\partial_{0,1} \circ\left(i_{p} \circ \partial_{p}^{p-2}+i_{p-1} \circ \partial_{p-1}^{p-2}\right)=\partial_{p} \circ \partial_{p}^{p-2}+\partial_{p-1} \circ \partial_{p-1}^{p-2},
$$

which finally finishes the proof.

We now want to consider an arbitrary permutation $\varphi:\{0, \ldots, p\} \rightarrow\{0, \ldots, p\}$. We shall denote by the same letter the unique affine isomorphism $\Delta^{p} \stackrel{\cong}{\longrightarrow} \Delta^{p}$ which is induced by applying $\varphi$ on the vertices. Let

$$
\Psi_{\varphi}: A\left(\Delta^{p}\right) \rightarrow \varphi^{*} A\left(\Delta^{p}\right)
$$

be the isomorphism of [10], Lemma 1.3. It clearly restricts to an isomorphism, also denoted $\Psi_{\varphi}$, between the ideals $A\left(\stackrel{\circ}{\Delta}^{p}\right)$ and $\varphi^{*} A\left(\stackrel{\circ}{\Delta}^{p}\right)$. We then get

Proposition 4.8. Let $\operatorname{sign}(\varphi)$ denote the sign of the permutation $\varphi:\{0, \ldots, p\} \rightarrow$ $\{0, \ldots, p\}$. Then the following diagram commutes:

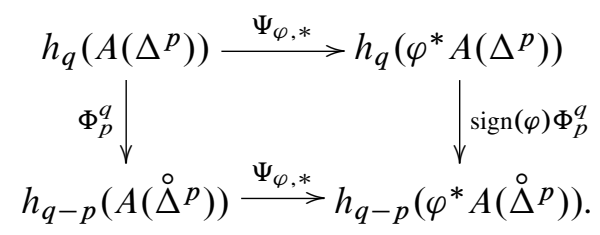


Proof. Since every permutation is a product of transpositions which interchange two neighbours in $\{0, \ldots, p\}$, we may assume without loss of generality that $\varphi$ interchanges $l$ with $l+1$.

If we identify $A\left(\Delta^{p}\right)$ with $\varphi^{*} A\left(\Delta^{p}\right)$ via $\Psi_{\varphi}$ and write $\Delta_{\varphi}^{l}$ for the $l$-dimensional face $\left\langle\varphi\left(v_{0}\right), \ldots, \varphi\left(v_{l}\right)>\subseteq \Delta^{p}\right.$, the above diagram restricts to showing that the isomorphism given by the composition

$$
\begin{aligned}
h_{q}\left(A\left(\Delta^{p}\right)\right) \stackrel{\mathrm{ev}_{\varphi(0)}, *}{\longrightarrow} h_{q}\left(A_{\varphi\left(v_{0}\right)}\right) & \rightarrow h_{q-1}\left(A\left(\stackrel{\circ}{\Delta}_{\varphi}^{1}\right)\right) \\
& \rightarrow h_{q-2}\left(A\left(\stackrel{\circ}{\Delta}_{\varphi}^{2}\right)\right) \rightarrow \cdots \rightarrow h_{q-p}\left(A\left(\stackrel{\circ}{\Delta}^{p}\right)\right)
\end{aligned}
$$

differs from the canonical isomorphism $\Phi_{q}^{p}: h_{q}\left(A\left(\Delta^{p}\right)\right) \rightarrow h_{q+p}\left(A\left(\AA^{p}\right)\right)$ by $\operatorname{sign}(\varphi)$. For this we first remark that by Lemma 4.3 we have the equality

$$
\Phi_{v_{0}, \varphi\left(v_{0}\right)} \circ \mathrm{ev}_{v_{0}, *}=\operatorname{ev}_{\varphi\left(v_{0}\right), *}: h_{q}\left(A\left(\Delta^{p}\right)\right) \rightarrow h_{q}\left(A_{\varphi\left(v_{0}\right)}\right),
$$

which then implies that we have to prove that the isomorphisms

$$
\Theta: h_{q}\left(A_{v_{0}}\right) \cong h_{q-1}\left(A\left(\stackrel{\circ}{\Delta}^{1}\right)\right) \cong h_{q-2}\left(A\left(\stackrel{\circ}{\Delta}^{2}\right)\right) \cong \cdots \cong h_{q-p}\left(A\left(\stackrel{\circ}{\Delta}^{p}\right)\right)
$$

and

$$
\Theta_{\varphi}: h_{q}\left(A_{\varphi\left(v_{0}\right)}\right) \cong h_{q-1}\left(A\left(\stackrel{\circ}{\Delta}_{\varphi}^{1}\right)\right) \cong h_{q-2}\left(A\left(\stackrel{\circ}{\Delta}_{\varphi}^{2}\right)\right) \cong \cdots \cong h_{q-p}\left(A\left(\stackrel{\circ}{\Delta}^{p}\right)\right)
$$

are related via

$$
\Theta=\operatorname{sign}(\varphi) \Theta_{\varphi} \circ \Phi_{v_{0}, \varphi\left(v_{0}\right)} .
$$

If $\varphi$ permutes 0 with 1 , then we have $\Delta_{\varphi}^{j}=\Delta^{j}$ for all $j \geq 1$, so the above equation reduces to the case $p=1$. This case is taken care for by Lemma 4.6 above. If $\varphi$ permutes $l$ with $l+1$ for some $l>0$, then we have $\Delta_{\varphi}^{j}=\Delta^{j}$ for all $j \geq l+1$, so we may assume without loss of generality that $l=p-1$. We then also have $\Delta_{\varphi}^{p-2}=\Delta^{p-2}$ and all we have to show is that the compositions

$$
\left.h_{r}\left(A\left(\stackrel{\circ}{\Delta}^{p-2}\right)\right) \cong h_{r-1}\left(A\left(\stackrel{\circ}{\Delta}_{p}^{p-1}\right)\right) \cong h_{r-2}\left(\stackrel{\circ}{\Delta}^{p}\right)\right)
$$

and

$$
\left.h_{r}\left(A\left(\stackrel{\circ}{\Delta}^{p-2}\right)\right) \cong h_{r-1}\left(A\left(\stackrel{\circ}{\Delta}_{p-1}^{p-1}\right)\right) \cong h_{r-2}\left(\stackrel{\circ}{\Delta}^{p}\right)\right)
$$

differ by the factor -1 . But this is shown in Lemma 4.7. This completes the proof.

In order to state the following important corollary, let us note that if $A\left(\Delta^{p}\right)$ is an $h_{*}$-fibration and if $\Delta_{l}^{p-1}$ is a face of $\Delta^{p}$, then the quotient map $A\left(\Delta^{p}\right) \rightarrow A\left(\Delta_{l}^{p-1}\right)$ induces an isomorphism

$$
\operatorname{res}_{l}: h_{*}\left(A\left(\Delta^{p}\right)\right) \rightarrow h_{*}\left(A\left(\Delta_{l}^{p-1}\right)\right),
$$

which follows from the simple fact that evaluation at any common vertex induces an isomorphism in $h_{*}$-theory for both algebras. With this notation we now get 
Corollary 4.9. Suppose that $A\left(\Delta^{p}\right)$ is an $h_{*}$-fibration and let

$$
\partial_{l}: h_{q-p+1}\left(A\left(\stackrel{\circ}{\Delta}_{l}^{p-1}\right)\right) \rightarrow h_{q-p}\left(A\left(\stackrel{\circ}{\Delta}^{p}\right)\right)
$$

denote the isomorphism of Definition 4.5. Let $\Phi_{p}^{q}: h_{q}\left(A\left(\Delta^{p}\right)\right) \rightarrow h_{q-p}\left(A\left(\stackrel{\diamond}{\Delta}^{p}\right)\right)$ denote the canonical isomorphism and let

$$
\Phi_{p-1}^{q}: h_{q}\left(A\left(\Delta_{l}^{p-1}\right)\right) \rightarrow h_{q-p+1}\left(A\left(\stackrel{\circ}{l}_{l}^{p-1}\right)\right)
$$

be the canonical isomorphism for $A\left(\Delta_{l}^{p-1}\right)$ with respect to the orientation of $\Delta_{l}^{p-1}$ inherited from $\Delta^{p}$. Then

$$
\partial_{l} \circ \Phi_{p-1}^{q} \circ \operatorname{res}_{l}=(-1)^{p-l} \Phi_{p}^{q} .
$$

Proof. Consider the permutation $\varphi:\{0, \ldots, p\} \rightarrow\{0, \ldots, p\}$ defined by

- $\varphi(k)=k$ for $k=0, \ldots, l-1$;

- $\varphi(k)=k+1$ for $k=l, \ldots, p-1$;

- $\varphi(p)=l$.

Then $\operatorname{sign}(\varphi)=(-1)^{p-l}$. If we identify $\varphi^{*} A$ with $A$ via $\Phi_{\varphi}$, as done in the proof of Proposition 4.8, then the isomorphism $\Phi_{p}^{q}$ for the fibration $\varphi^{*} A\left(\Delta^{p}\right)$ just becomes $\partial_{l} \circ \Phi_{p-1}^{q}$. By Proposition 4.8 this differs from the isomorphism $\Phi_{p}^{q}$ for $A\left(\Delta^{p}\right)$ by the factor $\operatorname{sign}(\varphi)=(-1)^{p-l}$.

The Leray-Serre spectral theorem. We are now going back to the situation of Proposition 4.1 in the special case where $X$ is a finite dimensional simplicial complex. In what follows, we write $C_{p}$ for the set of oriented closed $p$-simplexes in $X$. To be more precise, let us consider any element $\sigma \in C_{p}$ as a given affine realization $\sigma: \Delta^{p} \rightarrow \Delta_{\sigma}^{p} \subseteq X$ of the closed $p$-cell $\Delta_{\sigma}^{p}$ of $X$, which then induces an orientation on $\Delta_{\sigma}^{p}$. If $\sigma \in C_{p}$, we write $\sigma_{l}: \Delta_{l}^{p-1} \rightarrow \Delta_{\sigma, l}^{p-1} \subseteq X$ for the $l$-th face of $\sigma$. Then there exists a unique element $\tau \in C_{p-1}$ such that $\tau\left(\Delta^{p-1}\right)=\sigma_{l}\left(\Delta_{l}^{p-1}\right)$ and then a unique affine transformation $\varphi_{\sigma, \tau}^{l}: \Delta^{p-1} \rightarrow \Delta^{p-1} \cong \Delta_{l}^{p-1}$ such that

$$
\tau=\sigma_{l} \circ \varphi_{\sigma, \tau}^{l}
$$

These give precisely the gluing data for our simplicial complex $X$.

As outlined in Remark 4.2, under suitable finiteness conditions explained there, the $E_{1}$-terms are then given by

$$
E_{1}^{p, q}=\bigoplus_{\sigma \in C_{p}} h_{q}\left(A\left(\stackrel{\circ}{\triangle}_{\sigma}^{p}\right)\right)
$$


and, applied to the direct summand $h_{q+1}\left(A\left({\stackrel{\circ}{\Delta_{\tau}^{p-1}}}^{p-1}\right)\right.$ of $E_{1}^{p-1, q+1}$, the differential $d: E_{1}^{p-1, q+1} \rightarrow E_{1}^{p, q}$ projects to the direct summand $h_{q}\left(A\left(\stackrel{\circ}{\Delta}_{\sigma}^{p}\right)\right)$ of $E_{1}^{p, q}$ via the chain of maps

$$
\begin{aligned}
h_{q+1}\left(A\left(\stackrel{\circ}{\Delta}_{\tau}^{p-1}\right)\right) \stackrel{I_{q+1}^{p-1}(\tau)}{\longrightarrow} & h_{q+1}\left(A_{p-1, p-2}\right) \\
\stackrel{\iota *}{\longrightarrow} & h_{q+1}\left(A_{p-1}\right) \stackrel{\partial}{\rightarrow} h_{q}\left(A_{p, p-1}\right) \stackrel{Q_{q}^{p}(\sigma)}{\longrightarrow} h_{q}\left(A\left(\stackrel{\circ}{\Delta}_{\sigma}^{p}\right)\right),
\end{aligned}
$$

where as before, $A_{p}=A\left(X_{p}\right), A_{p, p-1}=A\left(X_{p} \backslash X_{p-1}\right) \cong \bigoplus_{\sigma \in C_{p}} A\left(\stackrel{\circ}{\Delta}_{\sigma}^{p}\right)$, $I^{p-1}(\tau): A\left(\stackrel{\circ}{\Delta}_{\tau}^{p-1}\right) \rightarrow A_{p-1, p-2}$ is the inclusion map corresponding to the open cell $\stackrel{\circ}{\Delta}_{\sigma}^{p}$ and $Q^{p}(\sigma): A_{p, p-1} \rightarrow A\left(\stackrel{\circ}{\Delta}_{\sigma}^{p}\right)$ is the quotient map. We now apply the inverses of the canonical isomorphisms

$$
\Phi_{p+q}^{p}: h_{p+q}\left(A\left(\Delta_{\sigma}^{p}\right)\right) \rightarrow h_{q}\left(A\left(\stackrel{\circ}{\sigma}_{\sigma}^{p}\right)\right)
$$

(see Definition 4.5) to each simplex $\sigma \in C_{p}$ (and similarly for $\tau \in C_{p-1}$ ), which gives us isomorphisms

$$
E_{1}^{p, q} \cong \bigoplus_{\sigma \in C_{p}} h_{p+q}\left(A\left(\Delta_{\sigma}^{p}\right)\right) \quad \text { and } \quad E_{1}^{p-1, q+1} \cong \bigoplus_{\tau \in C_{p-1}} h_{p+q}\left(A\left(\Delta_{\tau}^{p-1}\right)\right)
$$

In this picture, the differential is described on the summands via

$$
\begin{aligned}
d_{\tau}^{\sigma}: h_{p+q}\left(A\left(\Delta_{\tau}^{p-1}\right)\right) & \stackrel{\Phi_{p+q}^{p-1}}{\longrightarrow} h_{q+1}\left(A\left(\stackrel{\circ}{\Delta}_{\tau}^{p-1}\right)\right) \\
& \stackrel{I_{q+1}^{p-1}(\tau)}{\longrightarrow} h_{q+1}\left(A_{p-1, p-2}\right) \stackrel{\iota_{*}}{\longrightarrow} h_{q+1}\left(A_{p-1}\right) \stackrel{\partial}{\rightarrow} h_{q}\left(A_{p, p-1}\right) \\
& \stackrel{Q_{q}^{p}(\sigma)}{\longrightarrow} h_{q}\left(A\left(\stackrel{\circ}{\Delta}_{\sigma}^{p}\right)\right) \stackrel{\left(\Phi_{q+p}^{p}\right)^{-1}}{\longrightarrow} h_{p+q}\left(A\left(\Delta_{\sigma}^{p}\right)\right) .
\end{aligned}
$$

We then show

Proposition 4.10. Suppose that $A(X)$ is an $h_{*}$-fibration over the finite dimensional simplicial complex $X$. Then the map $d_{\tau}^{\sigma}$ in $(4.8)$ is zero if $\Delta_{\tau}^{p-1}$ is not a face of $\Delta_{\sigma}^{p}$, and we have

$$
d_{\tau}^{\sigma}=(-1)^{p-l} \operatorname{sign}\left(\varphi_{\sigma, \tau}^{l}\right)\left(\operatorname{res}_{\tau}^{\sigma}\right)^{-1}
$$

if $\Delta_{\tau}^{p-1}=\Delta_{\sigma, l}^{p-1}$, where we denote by $\operatorname{res}_{\tau}^{\sigma}: h_{*}\left(A\left(\Delta_{\sigma}^{p}\right)\right) \stackrel{\cong}{\longrightarrow} h_{*}\left(A\left(\Delta_{\tau}^{p-1}\right)\right)$ the isomorphism induced by the quotient map $A\left(\Delta_{\sigma}^{p}\right) \rightarrow A\left(\Delta_{\tau}^{p}\right)$.

For the proof of the proposition, we need the following lemma: 
Lemma 4.11. Suppose that $\sigma \in C_{p}$ and $\tau \in C_{p-1}$ are as above. Suppose further that $Z$ is any closed simplicial subcomplex of $X_{p}$ such that $\Delta_{\tau}^{p-1}$ and $\Delta_{\sigma}^{p}$ are contained in $Z$. Then the differential $d_{\tau}^{\sigma}$ of (4.8) coincides with the same map if we replace $X$ by $Z$.

Proof. Apply the quotient map $q: A(X) \rightarrow A(Z)$ to all ingredients of the composition of maps in (4.7) and use naturality of the long exact sequences in $h_{*}$-theory. Since the quotient map induces the identity in the first and in the last place of that chain, and since, by naturality, all other maps are linked by commutative diagrams (note that all maps in the chain are maps taken from appropriate long exact sequences linked by factorizations of the quotient map $A(X) \rightarrow A(Z)$ ), the result follows.

Proof of Proposition 4.10. Suppose first that $\Delta_{\tau}^{p-1}$ is not a face of $\Delta_{\sigma}^{p}$. To see that $d_{\tau}^{\sigma}$ is then the zero map, we actually show that the chain of maps of (4.7) relative to the subcomplex $Z=\Delta_{\tau}^{p-1} \cup \Delta_{\sigma}^{p}$ is the zero map. We then have $A\left(Z_{p-1}\right)=$ $A\left(\Delta_{\tau}^{p-1}\right) \oplus A\left(\Delta_{\sigma}^{p} \backslash \stackrel{\circ}{\Delta}_{\sigma}^{p}\right)$, and the chain of maps in (4.7) becomes the composition

$$
h_{q+1}\left(A\left(\stackrel{\circ}{\Delta}_{\tau}^{p-1}\right)\right) \stackrel{\iota_{*}^{*}}{\rightarrow} h_{q+1}\left(A\left(\Delta_{\tau}^{p-1}\right)\right) \oplus h_{q+1}\left(A\left(\Delta_{\sigma}^{p} \backslash \stackrel{\circ}{\Delta}_{\sigma}^{p}\right)\right) \stackrel{\partial}{\rightarrow} h_{q}\left(A\left(\stackrel{\circ}{\Delta}_{\sigma}^{p}\right)\right) .
$$

But the first map takes its image in the first summand of the middle term, which lies in the kernel of the second map.

So we can now restrict to the case where $\Delta_{\tau}^{p}$ coincides with the $l$-th face of $\Delta_{\sigma}^{p}$. By Lemma 4.11, we may also assume without loss of generality that $X=\Delta_{\sigma}^{p}$. Using once again naturality of long exact sequences in $h_{*}$-theory, and applying this to the inclusion of the ideal $A\left(\stackrel{\circ}{\Delta}_{\sigma, l}^{p-1} \cup \stackrel{\circ}{\Delta}_{\sigma}^{p}\right)$ into $A\left(\Delta_{\sigma}^{p} \backslash X_{p-2}\right)$ (which is the restriction to the complement of the $(p-2)$-skeleton of $\left.\Delta_{\sigma}^{p}\right)$ shows that in this situation the chain of maps in (4.7) reduces to the boundary map $\partial_{l}$ in the long exact sequence

$$
\rightarrow h_{q+1}\left(A\left(\stackrel{\circ}{\Delta}_{\sigma, l}^{p-1} \cup \stackrel{\circ}{\Delta}_{\sigma}^{p}\right)\right) \rightarrow h_{q+1}\left(A\left(\stackrel{\circ}{\Delta}_{\sigma, l}^{p-1}\right)\right) \stackrel{\partial_{l}}{\rightarrow} h_{q}\left(A\left(\stackrel{\circ}{\Delta}_{\sigma}^{p}\right)\right) \rightarrow .
$$

Moreover, it follows from Proposition 4.8, that replacing the orientation of $\Delta_{\tau}^{p-1}=$ $\Delta_{\sigma, l}^{p-1}$ given by $\tau$ to that given by $\sigma_{l}$ results to the factor $\operatorname{sign}\left(\varphi_{\sigma, \tau}^{l}\right)$ in the canonical oriented isomorphism $\Phi_{p+q}^{p-1}: h_{p+q}\left(A\left(\Delta_{\tau}^{p-1}\right)\right) \rightarrow h_{q}\left(A\left(\stackrel{\circ}{\tau}_{\tau}^{p-1}\right)\right)$. Taking this into account, the map $d_{\tau}^{\sigma}$ becomes $\operatorname{sign}\left(\varphi_{\sigma, \tau}^{l}\right)$-times the composition

$h_{p+q}\left(A\left(\Delta_{\sigma, l}^{p-1}\right)\right) \stackrel{\Phi_{p+q}^{p-1}}{\longrightarrow} h_{q+1}\left(A\left(\stackrel{\circ}{\triangle}_{\sigma, l}^{p-1}\right)\right) \stackrel{\partial_{l}}{\longrightarrow} h_{q}\left(A\left(\stackrel{\circ}{\Delta}_{\sigma}^{p}\right)\right) \stackrel{\left(\Phi_{p+q}^{p}\right)^{-1}}{\longrightarrow} h_{p+q}\left(A\left(\Delta_{\sigma}^{p}\right)\right)$.

But it follows from Corollary 4.9 that this composition coincides with $(-1)^{l}\left(\operatorname{res}_{\tau}^{\sigma}\right)^{-1}$ (which plays the role of res ${ }_{l}$ in that corollary). Hence we arrived at the equation

$$
d_{\tau}^{\sigma}=\operatorname{sign}\left(\varphi_{\sigma, \tau}^{l}\right)(-1)^{p-l}\left(\operatorname{res}_{\tau}^{\sigma}\right)^{-1},
$$

which finishes the proof. 
We now recall the definition of the simplicial cohomology of a finite dimensional and locally finite simplicial complex $X$ with coefficients in a group bundle $\mathcal{E}=$ $\bigcup\left\{G_{x} \mid x \in X\right\}$. We refer to Section 3 for the definition of a group bundle and for the notion of the group bundle $\mathscr{H}_{*}$ associated to an $h_{*}$-fibration.

If $Y$ is any simply connected subset of $X$, a section $g \in \Gamma(Y, \mathscr{E})$ is said to be constant if $g$ becomes constant in any trivialization of the bundle over $Y$, which is equivalent to saying that

$$
g(y)=\Phi_{x, y} g(x) \quad \text { for all } x, y \in Y .
$$

We denote by $\Gamma_{\text {const }}(Y, \mathcal{G})$ the group of constant sections of $Y$. It is clear that if $Y$ is simply connected and $Z \subseteq Y$ is any (simply connected) subset, then the restriction map

$$
\operatorname{res}_{Z}^{Y}: \Gamma_{\text {const }}(Y, \mathscr{E}) \rightarrow \Gamma_{\text {const }}(Z, \mathscr{E})
$$

is an isomorphism. In particular, $\Gamma_{\text {const }}(Y, \mathscr{E})$ is isomorphic to $G_{x}$ for every $x \in Y$.

We now define the $p$-cochains for simplicial cohomology on $X$ with coefficient $\mathcal{E}$ as

$$
C^{p}(X ; \mathcal{G}):=\left\{C_{p} \ni \sigma \mapsto f(\sigma) \in \Gamma_{\text {const }}\left(\Delta_{\sigma}^{p} ; \mathcal{E}\right)\right\},
$$

i.e., as the set of all maps which assign a $p$-simplex $\sigma \in C_{p}$ to a constant section on $\Delta_{\sigma}^{p}$. Moreover, we define $C_{\text {fin }}^{p}(X ; \mathcal{E})$ as the subgroup of $C^{p}(X ; \mathcal{E})$ consisting of all finitely supported functions. The boundary map

$$
\text { ə: } C^{p-1}(X ; \mathcal{E}) \rightarrow C^{p}(X ; \mathcal{E})
$$

is defined by

$$
\partial f(\sigma)=\sum_{l=0}^{p}(-1)^{l}\left(\operatorname{res}_{\sigma_{l}}^{\sigma}\right)^{-1}\left(f\left(\sigma_{l}\right)\right),
$$

where we put $f\left(\sigma_{l}\right)=\operatorname{sign}\left(\varphi_{\sigma, \tau}^{l}\right) f(\tau), \tau \in C_{p-1}$ is the unique element with $\Delta_{\tau}^{p-1}=$ $\Delta_{\sigma, l}^{p-1}$, and $\varphi_{\sigma, \tau}^{l}$ is defined as in (4.6). It restricts to a boundary map on $C_{\text {fin }}^{p}(X ; \mathscr{E})$. We define $H^{p}(X ; \mathcal{G})$ as the $p$-th cohomology of the chain complex $\left(C^{p}(X ; \mathcal{G}), \partial\right)$ and $H_{\text {fin }}^{p}(X ; \mathscr{E})$ as the $p$-th cohomology of $\left(C_{\text {fin }}^{p}(X ; \mathscr{E}), \partial\right)$. Of course, both cohomology groups coincide on finite complexes. The computations in Proposition 4.10 now immediately give

Theorem 4.12 (Leray-Serre spectral sequence for $h_{*}$-fibrations). Let $h_{*}$ be a homology theory on a good category of $C^{*}$-algebras and suppose that $X$ is a finite dimensional $\sigma$-finite and locally finite simplicial complex. Suppose that $A=A(X)$ is an $h_{*}$-fibration with associated group bundle $\mathscr{H}_{*}$. If $h_{*}$ is $\sigma$-additive or if $X$ is finite, the $E_{2}$-term in the spectral sequence of Proposition 4.1 is given by

$$
E_{2}^{p, q}=H_{\text {fin }}^{p}\left(X ; \mathscr{H}_{p+q}\right) .
$$


If $h_{*}$ is $\sigma$-multiplicative, then

$$
E_{2}^{p, q}=H^{p}\left(X ; \mathscr{H}_{p+q}\right) .
$$

Remark 4.13. (1) As mentioned earlier, the main example we have in mind for the above theorem is the case where $h_{*}$ is the K-theory functor. But the result applies also to other interesting functors such as the functor $\operatorname{KK}(B, \cdot)$ for a fixed $\mathrm{C}^{*}$-algebra $B$. In general, these functors are only finitely additive, so we should restrict to finite simplicial complexes in these situations.

(2) We should note that the cohomology groups $H^{p}(X ; \boldsymbol{E})$ we defined above coincide with the usual singular cohomology with local coefficients as defined in many standard text books (e.g., see [7]), while the groups $H_{\text {fin }}^{p}(X ; \mathcal{E})$ are known as the simplicial cohomology with local coefficients with finite supports.

Although we do not want to go through all the details for the proof of the LeraySerre spectral sequence for cohomology theories on $\mathrm{C}^{*}$-algebras (the steps are similar as for homology theory with all arrows reversed) we want at least give a proper statement of the result. As mentioned earlier, K-homology serves as a main example of such theory, but other examples are given by the functors $\mathrm{KK}(\cdot, B)$ for a fixed C*-algebra $B$.

Recall that the simplicial homology $H_{p}(X ; \mathcal{G})$ with coefficient in a group bundle $\mathcal{E}$ is defined as the homology of the chain complex $\left(C_{p}(X ; \mathcal{G}), d\right)$, where

$$
C_{p}(X ; \mathcal{E}):=\left\{\sum_{\sigma \in C_{p}} a_{\sigma} \sigma \mid a_{\sigma} \in \Gamma_{\text {const }}\left(\Delta_{\sigma}^{p} ; \mathcal{E}\right)\right\}
$$

with boundary map $d: C_{p}(X ; \mathcal{G}) \rightarrow C_{p-1}(X ; \mathcal{G})$ given by

$$
d\left(a_{\sigma} \sigma\right)=\sum_{l=0}^{p}(-1)^{l} \operatorname{sign}\left(\varphi_{\sigma, \tau}^{l}\right) \operatorname{res}_{\sigma_{i}}^{\sigma}\left(a_{\sigma}\right) \tau_{l},
$$

where for each $0 \leq l \leq p, \tau_{l}$ is the unique element in $C_{p-1}$ with image $\Delta_{\sigma, l}^{p-1}$ and $\operatorname{sign}\left(\varphi_{\sigma, \tau}^{l}\right)$ is as before. Again, if we restrict to finite sums, we obtain the theory $H_{p}^{\text {fin }}(X ; \mathcal{E})$ with finite supports. The Leray-Serre theorem then reads as follows.

Theorem 4.14 (Leray-Serre spectral sequence for $h^{*}$-fibrations). Let $h^{*}$ be a cohomology theory on a good category of $C^{*}$-algebras and let $X$ be as in Theorem 4.12. Suppose that $A=A(X)$ is an $h^{*}$-fibration with associated group bundle $\mathscr{H}^{*}$. If $h^{*}$ is $\sigma$-additive or if $X$ is finite, the $E^{2}$-term in the spectral sequence of Proposition 4.1 is given by

$$
E_{p, q}^{2}=H_{p}^{\mathrm{fin}}\left(X ; \mathscr{H}_{p+q}\right)
$$

If $h^{*}$ is $\sigma$-multiplicative, then

$$
E_{p, q}^{2}=H_{p}\left(X ; \mathscr{H}_{p+q}\right)
$$


We want to close this section with a discussion how the spectral sequences considered here give new invariants for $\mathcal{R K K}$-equivalence of $\mathrm{C}^{*}$-algebra bundles.

The following lemma might be well known to the experts, but since we rely heavily on it, we give the argument here. For notation, we let $\mathcal{K} \mathcal{K}$ denote the category whose objects are separable $C^{*}$-algebras and the morphisms between two objects $A$ and $B$ are the elements in $\operatorname{KK}(A, B)$. Recall that for any pair of bundles $A(X)$ and $B(X)$ and any continuous inclusion map $f: Y \hookrightarrow X$ there exists a canonical pull-back map

$$
f^{*}: \mathcal{R K K}(X ; A(X), B(X)) \rightarrow \mathcal{R K K}\left(Y ; f^{*} A(Y), f^{*} B(Y)\right) .
$$

If $Y \subseteq X$, then we obtain restrictions $\underset{x}{\operatorname{x}} \mapsto \operatorname{res}_{Y}^{X}(\mathfrak{x})$ from $\mathcal{R K K}(X, A(X), B(X)) \rightarrow$ $\mathcal{R K K}(Y ; A(Y), B(Y))$ given via the inclusion of $Y$ into $X$ (see [17], Proposition 2.2). Recall also that a short exact extension $0 \rightarrow J \rightarrow A \stackrel{q}{\rightarrow} A / J \rightarrow 0$ of $\mathrm{C}^{*}$-algebras is semi-split if there exists a completely positive section $s: A / J \rightarrow A$. Note that this is always true if $A / J$ is nuclear (which follows if $A$ is nuclear).

Lemma 4.15. Suppose that $A(X)$ and $B(X)$ are $C^{*}$-algebra bundles over $X$ and suppose that $U \subset X$ is open. Let $x \in \mathcal{R K K}(X ; A, B)$ and let $i_{A}: A(U) \rightarrow A(X)$ and $q_{A}: A(X) \rightarrow A(X \backslash U)$ denote the inclusion and quotient maps (and similarly for $B(X)$ ), respectively. Then the diagram

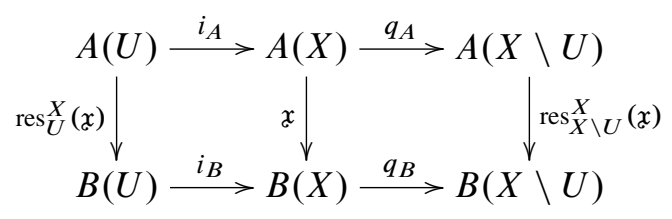

commutes in the category $\mathcal{K} \mathcal{K}$. Moreover, if both extensions in the above diagram are semi-split (we do not require that the completely positive sections are $C_{0}(X)$-linear), then the diagram

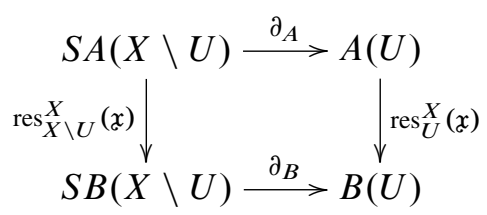

also commutes in $\mathcal{K} \mathcal{K}$, where $S A$ and $S B$ denote the suspensions of $A$ and $B$, respectively.

Proof. Let $(E(X), T)$ be a Kasparov cycle representing $x$. We may assume that $A(X)$ acts nondegenerately on $E(X)$. If $f: Y \rightarrow X$ is a continuous map, then $f^{*}(x)$ is represented by the cycle

$$
\left(C_{0}(Y) \otimes_{C_{0}(X)} E(X), 1 \otimes T\right) \cong\left(E(X) \otimes_{C_{0}(X)} C_{0}(Y), T \otimes 1\right),
$$


depending on whether we want to tensor $C_{0}(Y)$ from the left or from the right. The first square of the first diagram follows from an obvious isomorphism of $\mathrm{KK}(A(U), B(X))$ cycles

$$
\left(A(U) \otimes_{A(X)} E(X), 1 \otimes T\right) \cong\left(C_{0}(U) \otimes_{C_{0}(X)} E(X), 1 \otimes T\right) .
$$

The left cycle represents $\left[i_{A}\right] \otimes_{A(X)} x$ and the right cycle represents $\operatorname{res}_{U}^{X}(x) \otimes_{B(U)}\left[i_{B}\right]$.

Similarly, the second square of the first diagram follows from the observation that both products $\left[q_{A}\right] \otimes_{A(X \backslash U)} \operatorname{res}_{X \backslash U}^{X}(\mathfrak{x})$ and $\mathfrak{x} \otimes_{B(X)}\left[q_{B}\right]$ are represented by the module $\left(E(X) \otimes_{C_{0}(X)} C_{0}(X \backslash U), T \otimes 1\right)$ with the canonical module actions.

So let us now assume that both extensions are semi-split. Then the boundary map $\partial_{A}$ in the second diagram is given by Kasparov product with an element $\left[\partial_{A}\right] \in \mathrm{KK}_{1}(A(X \backslash U), A(U))$ constructed as follows: Let

$$
Z=((0,1] \times(X \backslash U)) \cup(\{1\} \times U) \subseteq(0,1] \times X
$$

Let $p: Z \rightarrow X$ denote the canonical projection and write $A(Z)$ for the pull-back $p^{*} A(Z)$. Note that, as an algebra, $A(Z)$ is just the mapping cone $C_{q_{A}}$ of the homomorphism $q_{A}$. Let $e_{A}: A(U) \rightarrow A(Z)$ be the inclusion map given by identifying $U$ with the open set $\{1\} \times U \subseteq Z$. It is shown in [2], Theorem 19.5.5, that $e_{A}$ is a KK-equivalence. Let $u_{A}$ denote its inverse and let $j_{A}: S A(X \backslash U)=$ $A((0,1) \times(X \backslash U)) \rightarrow A(Z)$ denote the inclusion. Then it is shown in [2], Theorem 19.5.7, that $\left[\partial_{A}\right]=\left[j_{A}\right] \otimes_{A(Z)} u_{A}$. The same construction applies to $B(X)$. Let $p^{*}(x)$ be the pull-back of $x$ in $\mathcal{R K K}(Z ; A(Z), B(Z))$. Then the commutativity of the second diagram follows from the commutativity of

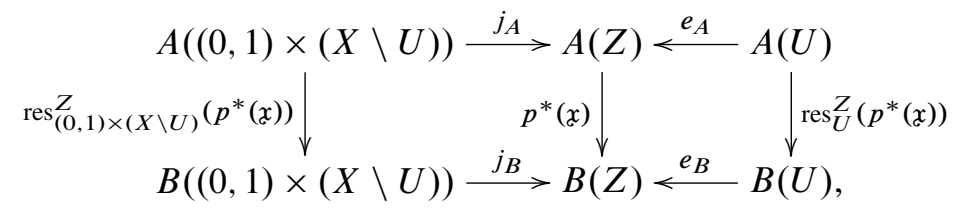

which is a consequence of the commutativity of the first diagram in the lemma.

We say that a (co-)homology theory on $\ell_{\text {sep }}$ is KK-representable if there exists a $\mathrm{C}^{*}$-algebra $B$ such that the (co-)homology theory is given by $A \mapsto \mathrm{KK}_{*}(B, A)$ (resp. $A \mapsto \mathrm{KK}(A, B)$ ). Of course $\mathrm{K}$-theory and $\mathrm{K}$-homology are important examples, but also $\mathrm{K}$-theory with coefficients in $\mathbb{Z} / n \mathbb{Z}$ is an example of such theory. Note that every KK-fibration is automatically an $h_{*}$-fibration (resp. $h^{*}$-fibration) if $h_{*}\left(\right.$ resp. $\left.h^{*}\right)$ is KK-representable.

Corollary 4.16. Let $h_{*}$ be a KK-representable homology theory on $\mathcal{C}_{\text {sep. Assume }}$ that $A(X)$ and $B(X)$ are $h_{*}$-fibrations. Then any class $\underset{x}{ } \in \mathcal{R K K}(X ; A, B)$ induces a morphism between the associated group bundles $\mathscr{H}_{*}(A)$ and $\mathscr{H}_{*}(B)$. If $X$ is a 
$C W$-complex and $A(X)$ and $B(X)$ are nuclear, then $X$ induces a morphism between the associated exact couples for the Leray-Serre spectral sequence.

In particular, if $x$ is an $\mathcal{R K K}$-equivalence, then it induces an isomorphism between the Leray-Serre spectral sequences for $h_{*}(A)$ and $h_{*}(B)$. A similar statement holds for KK-representable cohomology theories.

Remark 4.17. Note that in the case of K-theory one can omit the nuclearity assumption on $A(X)$ and $B(X)$ in the above lemma. The reason is that for $U \subseteq X$ open, and $Z$ as in the proof of Lemma 4.15 , we always have an isomorphism $\mathrm{K}_{*}(A(U)) \stackrel{e_{*}}{\cong}$ $\mathrm{K}_{*}(A(Z))$ such that the boundary map $\partial_{A}: K_{*+1}(A(X \backslash U)) \rightarrow \mathrm{K}_{*}(A(U))$ is given via the composition $\partial_{A}=\left(e_{*}\right)^{-1} \circ j_{A, *}$, with $e_{A}$ and $j_{A}$ as in the proof of the lemma. Thus the same argument as in the lemma shows that for each such $U$ the transformation given by Kasparov product with the appropriate restrictions of $x$ gives a transformation between the K-theory long exact sequences for $A$ and $B$ corresponding to $U$. This is all we need to obtain a well-defined morphism between the exact couples.

\section{Applications to noncommutative torus bundles}

Recall from [10] that a noncommutative principal $\mathbb{T}^{n}$-bundle (or NCP $\mathbb{T}^{n}$-bundle for short) is defined as a $\mathrm{C}^{*}$-algebra bundle $A(X)$ equipped with a fibrewise action of $\mathbb{T}^{n}$ such that $A(X) \rtimes \mathbb{T}^{n}$ is isomorphic to $C_{0}(X, \mathcal{K})$. By Takesaki-Takai duality, every such bundle can be realized up to stabilization by a crossed product $C_{0}(X, \mathcal{K}) \rtimes \mathbb{Z}^{n}$ for some fibrewise action of $\mathbb{Z}^{n}$ on $C_{0}(X, \mathcal{K})$. Using results from [11], [12] we showed in [10], §2, that the $\mathbb{T}^{n}$-equivariant stable isomorphism classes of NCP $\mathbb{T}^{n}$-bundles over a given space $X$ can be classified by the pairs $([Y], f)$, where $[Y]$ denotes the isomorphism class of a classical principal $\mathbb{T}^{n}$-bundle $Y \stackrel{q}{\rightarrow} X$ and $f: X \rightarrow \mathbb{T}^{\frac{n(n-1)}{2}}$ is a continuous map. The NCP $\mathbb{T}^{n}$-bundle corresponding to the pair $([Y], f)$ is then given by

$$
Y *\left(f^{*}\left(C^{*}\left(H_{n}\right)\right)\right)=\left(C_{0}(Y) \otimes_{C_{0}(X)} f^{*}\left(C^{*}\left(H_{n}\right)\right)\right)^{\mathbb{T}^{n}} .
$$

Let us recall the ingredients of this construction: $C^{*}\left(H_{n}\right)$ is the group $\mathrm{C}^{*}$-algebra of the group

$$
H_{n}=\left\langle g_{1}, \ldots, g_{n}, f_{i j}: 1 \leq i<j \leq n\right\rangle
$$

with relations $g_{i} g_{j}=f_{i j} g_{j} g_{i}$ and $f_{i j}$ central for all $1 \leq i<j \leq n$. This group has center $Z_{n}=\left\langle f_{i j}: 1 \leq i<j \leq n\right\rangle \cong \mathbb{Z}^{\frac{n(n-1)}{2}}$ and, therefore, $C^{*}\left(H_{n}\right)$ is a continuous $C^{*}$-algebra bundle over $\mathbb{T}^{\frac{n(n-1)}{2}} \cong \widehat{Z}_{n}$ via the inclusion

$$
\Phi: C\left(\mathbb{T}^{\frac{n(n-1)}{2}}\right) \cong C^{*}\left(Z_{n}\right) \rightarrow Z\left(C^{*}\left(H_{n}\right)\right) .
$$


Moreover, if we equip $C^{*}\left(H_{n}\right)$ with the dual action of $\mathbb{T}^{n} \cong \widehat{H_{n} / Z_{n}}$ it becomes an NCP $\mathbb{T}^{n}$-bundle over $\mathbb{T} \frac{n(n-1)}{2}$. We shall denote by $U_{1}, \ldots, U_{n}$ the unitaries of $C^{*}\left(H_{n}\right)$ corresponding to $g_{1}, \ldots, g_{n}$, respectively, and by $W_{i, j}$ the unitaries corresponding to $f_{i, j}$ for $1 \leq i<j \leq n$. If $f: X \rightarrow \mathbb{T}^{\frac{n(n-1)}{2}}$ is a continuous map, then the pull-back $f^{*}\left(C^{*}\left(H_{n}\right)\right)$ becomes an NCP $\mathbb{T}^{n}$-bundle over $X$. By taking the $C_{0}(X)$-balanced tensor product of $f^{*}\left(C^{*}\left(H_{n}\right)\right.$ ) with $C_{0}(Y)$ (with $C_{0}(X)$-action on $C_{0}(Y)$ induced by $q: Y \rightarrow X$ in the obvious way), and then taking the algebra of fixed points with respect to the diagonal action (with action by the inverse automorphism on one factor) provides the NCP $\mathbb{T}^{n}$-bundle $Y *\left(f^{*}\left(C^{*}\left(H_{n}\right)\right)\right)$. The $\mathbb{T}^{n}$-action is induced by the given $\mathbb{T}^{n}$-action on $Y$.

In [10] we studied the topological nature of the $\mathrm{C}^{*}$-algebra bundles $A(X)$ after "forgetting" the underlying $\mathbb{T}^{n}$-actions. In particular, we were interested in the question under what conditions two such bundles are K-theoretically equivalent fibrations, i.e., when are two such bundles $\mathcal{R K K}$-equivalent. We arrived at the following result:

Theorem 5.1 ([10], Theorem 7.2). Let $A(X)$ be a NCP $\mathbb{T}^{n}$-bundle over the path connected space $X$ and let $f: X \rightarrow \mathbb{T}^{\frac{n(n-1)}{2}}$ be the continuous map associated to $A(X)$ as above. Then the following statements are equivalent:

(i) $f$ is homotopic to a constant map.

(ii) The $K$-theory bundle $\mathcal{K}_{*}(A(X))$ is trivial.

(iii) $A(X)$ is $\mathcal{R K K}$-equivalent to $C_{0}(Y)$ for some (commutative) principal $\mathbb{T}^{n}$-bundle $q: Y \rightarrow X$.

In this section we will use the Leray-Serre spectral sequence to obtain the following triviality result.

Theorem 5.2. Let $A(X)$ be the NCP $\mathbb{T}^{n}$-bundle corresponding to the pair $([Y], f)$ as explained above such that $X$ is a finite dimensional locally finite and $\sigma$-finite simplicial complex. Then the following statements are equivalent:

(i) $A(X)$ is $\mathcal{R K K}$-equivalent to $C_{0}\left(X \times \mathbb{T}^{n}\right)$.

(ii) The $K$-theory bundle $\mathcal{K}_{*}(A)$ is trivial and all $d_{2}$-differentials in the Leray-Serre spectral theorem for $A(X)$ vanish.

(iii) $f$ is homotopic to a constant map and $Y \cong X \times \mathbb{T}^{n}$ as $\mathbb{T}^{n}$-bundles.

The proof depends on explicit calculations of the $d_{2}$ maps in the spectral sequence of a commutative principal $\mathbb{T}^{n}$-bundle $q: Y \rightarrow X$, and then transporting this result to the spectral sequence for the $\mathrm{K}_{*}$-fibration $A(X)$. In what follows we shall always denote by $Y_{x}$ the fibre of a given principal $\mathbb{T}^{n}$-bundle over a point $x \in X$ and we write $\left\{E_{r}^{p, q}(Y), d_{r}\right\}$ for the spectral sequence corresponding to a fixed triangulation 
of the base $X$. We always assume that $X$ is finite dimensional and $\sigma$-finite. The following proposition is certainly well known to the experts, but since we did not find an appropriate reference we give a proof.

Proposition 5.3. Let $Y \stackrel{q}{\rightarrow} X$ be a principal $\mathbb{T}$-bundle. Then the $(0,1)$-degree component of the differential

$$
d_{2}^{0,1}: H^{0}\left(X, \mathrm{~K}^{1}\left(Y_{x}\right)\right) \rightarrow H^{2}\left(X, \mathrm{~K}^{0}\left(Y_{x}\right)\right)
$$

on $E_{2}^{*, *}(Y)$ vanishes if and only if $Y \stackrel{q}{\rightarrow} X$ is trivial.

The proof of this proposition will require some preliminary work. Let $X_{-1}=$ $\emptyset \subset X_{0} \subset \cdots \subset X_{n}=X$ be the skeleton decomposition of $X$ and let us set $Y_{k}=q^{-1}\left(X_{k}\right)$. For $\sigma$ a simplex of $X$, let us denote by $V_{\sigma}$ the closure of the $*$-neighbourhood of $\sigma$. We may assume that

- for all simplices $\sigma$ there exist continuous maps $\Psi_{\sigma}: q^{-1}\left(V_{\sigma}\right) \rightarrow \mathbb{T}$,

- for all pairs of simplices $\sigma$ and $\sigma^{\prime}$ which are faces of a common simplex there exists a continuous map $h_{\sigma, \sigma^{\prime}}: V_{\sigma} \cap V_{\sigma^{\prime}} \rightarrow \mathbb{R}$,

such that

(i) $q^{-1}\left(V_{\sigma}\right) \rightarrow V_{\sigma} \times \mathbb{T} ; y \mapsto\left(q(y), \Psi_{\sigma}(y)\right)$ is a $\mathbb{T}$-equivariant homeomorphism,

(ii) $\Psi_{\sigma^{\prime}}=\Psi_{\sigma} \cdot e^{2 i \pi h_{\sigma, \sigma^{\prime}}}$ on $V_{\sigma} \cap V_{\sigma^{\prime}}$ for all pairs of simplices $\sigma$ and $\sigma^{\prime}$ which are faces of a common simplex.

We denote by $\mathcal{V}$ the corresponding atlas. Notice that this atlas provides an identification $\mathrm{K}^{1}\left(Y_{x}\right) \cong \mathrm{K}^{1}(\mathbb{T})$ induced by $Y_{x} \rightarrow \mathbb{T}, y \mapsto \Psi_{\sigma}(y)$, for any $x$ in the simplex $\sigma$. Although the identification $Y_{x} \cong \mathbb{T}$ depends on $\sigma$, it follows from (ii) that the induced map $\mathrm{K}^{1}\left(Y_{x}\right) \cong \mathrm{K}^{1}(\mathbb{T})$ does not.

Let $X_{0}=\left\{x_{0}, x_{1}, x_{2}, x_{3}, \ldots\right\}$ be the set of vertices of $X$. If $x_{i}$ and $x_{j}$ are connected by an edge, we denote by $e_{i, j}$ the oriented edge starting at $x_{i}$ and ending at $x_{j}$. For $t \in[0,1]$, we define $x_{i, j}(t)=(1-t) x_{i}+t x_{j}$ in $e_{i, j}$. Let $U_{\mathcal{V}}: Y_{0} \rightarrow \mathbb{T}$ be the continuous map such that $U_{\mathcal{V}}$ and $\Psi_{x_{i}}$ coincide on $q^{-1}\left(x_{i}\right)$. We extend $U_{\mathcal{V}}$ to a continuous map $W_{\mathcal{V}}: Y_{1} \rightarrow \mathbb{T}$ in the following way: If $x_{i}$ and $x_{j}$ are connected by the oriented edge $e_{i, j}$, we define $W_{\mathcal{V}}$ on $q^{-1}\left(e_{i, j}\right)$ by

$$
W_{\mathcal{V}}(z)=e^{2 i \pi t h_{x_{i}, x_{j}}(q(z))} \Psi_{x_{i}}(z)
$$

for $q(z)=x_{i, j}(t)$. We have

$$
\begin{aligned}
& E_{1}^{0,1}(Y)=\mathrm{K}^{1}\left(Y_{0} \backslash Y_{-1}\right)=\mathrm{K}^{1}\left(Y_{0}\right), \\
& E_{1}^{1,2}(Y)=\mathrm{K}^{0}\left(Y_{1} \backslash Y_{0}\right),
\end{aligned}
$$


and $d_{1}^{0,1}: \mathrm{K}^{1}\left(Y_{0}\right) \rightarrow \mathrm{K}^{0}\left(Y_{1} \backslash Y_{0}\right)$ is the boundary map $\partial$ associated to the pair $\left(Y_{0}, Y_{1}\right)$. Since $U_{\mathcal{V}}$ is the restriction of $W_{\mathcal{V}}$ to $Y_{0}$, the class $\left[U_{\mathcal{V}}\right]$ of $U_{\mathcal{V}}$ in $\mathrm{K}^{1}\left(Y_{0}\right)$ satisfies $\partial\left[U_{\mathcal{V}}\right]=0$ and $\left[U_{\mathcal{V}}\right]$ defines a class $\omega_{\mathcal{V}}$ in $E_{2}^{0,1}(Y) \cong H^{0}\left(X, \mathrm{~K}^{1}\left(Y_{x}\right)\right) \cong \mathbb{Z}$, which is thereby a generator.

Lemma 5.4. With the notation above and up to the canonical identification $\mathrm{K}^{0}\left(Y_{x}\right) \cong \mathbb{Z}$ (which sends [1] to 1$), d_{2}^{1,0} \omega \mathcal{V} \in H^{2}\left(X, \mathrm{~K}^{0}\left(Y_{x}\right)\right) \cong H^{2}(X, \mathbb{Z})$ is the first Chern class of $Y$.

Proof. We extend $W_{\mathcal{V}}: Y^{1} \rightarrow \mathbb{T}$ to a continuous map $\phi_{\mathcal{V}}: Y_{2} \rightarrow \mathbb{C}$ given on $Y_{\sigma}=q^{-1}(\sigma) \cong \sigma \times \mathbb{T}$ for a 2-simplex $\sigma$ in $X$ with boundary $\partial \sigma$ and center $x_{\sigma}$ by $\phi_{\mathcal{V}}\left(t x+(1-t) x_{\sigma}, z\right)=t W_{\mathcal{V}}(x, z)$ for $t$ in $[0,1], x$ in $\partial \sigma$ and $z \in \mathbb{T}$. Then

$$
V_{\mathcal{V}}:=\left(\begin{array}{cc}
\phi_{\mathcal{V}} & -\left(1-\phi_{\mathcal{V}} \bar{\phi}_{\mathcal{V}}\right)^{1 / 2} \\
\left(1-\phi_{\mathcal{V}} \bar{\phi}_{\mathcal{V}}\right)^{1 / 2} & \bar{\phi}_{\mathcal{V}}
\end{array}\right)
$$

is a lift in $U_{2}\left(C\left(Y_{2}\right)\right)$ for $\left(\begin{array}{cc}W_{\mathcal{V}} & 0 \\ 0 & \bar{W}_{\mathcal{V}}\end{array}\right)$ and thus $\partial\left[W_{\mathcal{V}}\right]=\left[P_{\mathcal{V}}\right]-\left[\left(\begin{array}{ll}1 & 0 \\ 0 & 0\end{array}\right)\right]$, where

$$
P_{\mathcal{V}}=V_{\mathcal{V}} \cdot\left(\begin{array}{ll}
1 & 0 \\
0 & 0
\end{array}\right) \cdot V_{\mathcal{V}}^{*}=\left(\begin{array}{cc}
\phi_{\mathcal{V}} \bar{\phi}_{\mathcal{V}} & \phi_{\mathcal{V}}\left(1-\phi_{\mathcal{V}} \bar{\phi}_{\mathcal{V}}\right)^{1 / 2} \\
\bar{\phi}_{\mathcal{V}}\left(1-\phi_{\mathcal{V}} \bar{\phi}_{\mathcal{V}}\right)^{1 / 2} & 1-\phi_{\mathcal{V}} \bar{\phi}_{\mathcal{V}}
\end{array}\right)
$$

is a projection in $M_{2}\left(C\left(Y_{2}\right)\right)$. Then, up to Bott periodicity, $d_{2}^{1,0} \omega$ is the class in $H^{2}\left(X, \mathrm{~K}^{0}\left(Y_{x}\right)\right)=E_{2}^{2,0}(Y)$ of the simplicial 2-cocycle $c$, with value on a 2-simplex $\sigma$ oriented by its boundary $\partial \sigma$

$$
c(\sigma)=i_{\sigma}^{*} \circ \partial\left[W_{\mathcal{\nu}}\right] \in \mathrm{K}^{0}\left(q^{-1}(\stackrel{\circ}{\sigma})\right) \cong \mathrm{K}^{0}(\stackrel{\circ}{\sigma} \times \mathbb{T}),
$$

where $\stackrel{\circ}{\sigma}=\sigma \backslash \partial \sigma$ and $i_{\sigma}$ is the inclusion map $i_{\sigma}: \stackrel{\circ}{\sigma} \hookrightarrow Y_{2} \backslash Y_{1}$. Finally, we get

$$
c(\sigma)=\left[P_{\mathcal{V}} \mid \sigma\right]-\left[\left(\begin{array}{ll}
1 & 0 \\
0 & 0
\end{array}\right)\right] \in \mathrm{K}^{0}\left(q^{-1}(\stackrel{\circ}{\sigma})\right),
$$

where $\left.P_{\mathcal{V}}\right|_{\sigma} \in M_{2}\left(C\left(q^{-1}(\sigma)\right)\right.$ is the restriction of $P_{\mathcal{V}}$ to $q^{-1}(\sigma)$. Let us denote by $\Phi_{\sigma}$ the inverse of the trivialisation map $q^{-1}(\sigma) \rightarrow \sigma \times \mathbb{T}, y \mapsto\left(q(y), \Psi_{\sigma}(y)\right)$. We then get isomorphisms

$$
\mathrm{K}^{0}\left(q^{-1}(\stackrel{\circ}{\sigma})\right) \cong \mathrm{K}^{0}(\stackrel{\circ}{\sigma}) \cong \mathbb{Z},
$$

where

- the first isomorphism is induced by $\stackrel{\circ}{\sigma} \rightarrow q^{-1}(\stackrel{\circ}{\sigma}), x \mapsto \Phi_{\sigma}(x, 1)$ (if we identify $q^{-1}(\stackrel{\circ}{\sigma})$ with $\stackrel{\circ}{\sigma} \times \mathbb{T}$ via $\Psi_{\sigma}$, this simply becomes $\stackrel{\circ}{\sigma} \rightarrow \stackrel{\circ}{\sigma} \times \mathbb{T}, x \mapsto(x, 1)$ );

- the second map is the Bott periodicity for the interior $\stackrel{\circ}{\sigma} \cong \mathbb{R}^{2}$ of the oriented simplex $\sigma$. 
Let us define for a continuous map $f: \sigma \rightarrow \mathbb{C}$ with $|f| \leq 1$ and $|f|=1$ on $\partial \sigma$ the projector

$$
P_{f}=\left(\begin{array}{cc}
|f|^{2} & f\left(1-|f|^{2}\right)^{1 / 2} \\
\bar{f}\left(1-|f|^{2}\right)^{1 / 2} & 1-|f|^{2}
\end{array}\right) .
$$

Then $\left[P_{f}\right]-\left[\left(\begin{array}{ll}1 & 0 \\ 0 & 0\end{array}\right)\right]$ is the image of $\left[\left.f\right|_{\partial \sigma}\right] \in \mathrm{K}^{1}(\partial \sigma)$ under the boundary map in $\mathrm{K}$-theory associated to the exact sequence

$$
0 \rightarrow C_{0}(\stackrel{\circ}{\sigma}) \rightarrow C(\sigma) \rightarrow C(\partial \sigma) \rightarrow 0 .
$$

In particular $\left[P_{f}\right]-\left[\left(\begin{array}{ll}1 & 0 \\ 0 & 0\end{array}\right)\right]$ only depends on the winding number of $\left.f\right|_{\partial \sigma}$ on $\partial \sigma$ and this winding number is precisely the image of $\left[P_{f}\right]-\left[\left(\begin{array}{ll}1 & 0 \\ 0 & 0\end{array}\right)\right]$ under the second isomorphism of eq. (5.1). Consequently, if we set

$$
f_{\mathcal{V}, \sigma}: \sigma \rightarrow \mathbb{C}, \quad x \mapsto \phi \mathcal{V} \circ \Phi_{\sigma}(x, 1),
$$

then the image of $c(\sigma)$ under the chain of isomorphism of eq. (5.1) is the winding number of the restriction of $f_{\mathcal{V}, \sigma}$ to the oriented boundary $\partial \sigma$.

If $\sigma$ has vertices $x_{i}, x_{j}$ and $x_{k}$ connected by oriented edges $e_{i, j}, e_{j, k}$ and $e_{k, i}$, then since $h_{\sigma, x_{i}}+h_{x_{i}, x_{j}}-h_{\sigma, x_{j}}$ and $h_{\sigma, x_{j}}+h_{x_{j}, x_{k}}-h_{\sigma, x_{k}}$ are integers, we have $f_{\mathcal{V}, \sigma}=e^{2 i \pi h_{\sigma, x_{i}}} \cdot g_{\mathcal{V}, \sigma}$, where

$$
\begin{aligned}
& g_{\mathcal{V}, \sigma}\left(x_{i, j}(t)\right)=\exp \left(2 i \pi t h_{x_{i}, x_{j}}\left(x_{i, j}(t)\right)\right), \\
& g_{\mathcal{V}, \sigma}\left(x_{j, k}(t)\right)=\exp \left(2 i \pi\left(h_{x_{i}, x_{j}}\left(x_{j, k}(t)\right)+t h_{x_{j}, x_{k}}\left(x_{j, k}(t)\right)\right)\right), \\
& g_{\mathcal{V}, \sigma}\left(x_{k, i}(t)\right)=\exp \left(2 i \pi\left(h_{x_{i}, x_{j}}\left(x_{k, i}(t)\right)+h_{x_{j}, x_{k}}\left(x_{k, i}(t)\right)+t h_{x_{k}, x_{i}}\left(x_{k, i}(t)\right)\right)\right) .
\end{aligned}
$$

But $e^{2 i \pi h_{\sigma, x_{i}}}$ has winding number 0 , and $h_{x_{i}, x_{j}}$ and $h_{x_{j}, x_{k}}$ can be pushed forward homotopically to the edge $e_{k, i}$, and thus the restriction of $g_{\mathcal{V}, \sigma}$ to $\partial \sigma$ is a unitary homotopic to

$$
\begin{aligned}
x_{i, j}(t) & \mapsto 1, \\
x_{j, k}(t) & \mapsto 1, \\
x_{k, i}(t) & \mapsto \exp \left(2 i \pi t\left(h_{x_{i}, x_{j}}\left(x_{k, i}(t)\right)+h_{x_{j}, x_{k}}\left(x_{k, i}(t)\right)+h_{x_{k}, x_{i}}\left(x_{k, i}(t)\right)\right)\right) .
\end{aligned}
$$

Since $h_{x_{i}, x_{j}}+h_{x_{j}, x_{k}}+h_{x_{k}, x_{i}}$ is an integer $m_{\sigma}$, the restriction to $\partial \sigma$ of $g_{\mathcal{V}, \sigma}$ and hence of $f_{\mathcal{V}, \sigma}$ has winding number $m_{\sigma}$. Up to the composition of the two isomorphisms of eq. (5.1), we finally get that $c(\sigma)=m_{\sigma}$, which is precisely the cocycle defining the first Chern class of $Y$.

Proof of Proposition 5.3. Since $\omega \mathcal{v}$ is a generator for $E_{2}^{0,1}(Y) \cong \mathbb{Z}$, we see from Lemma 5.4 that $d_{2}^{0,1}: E_{2}^{0,1}(Y) \rightarrow E_{2}^{2,0}(Y)$ is vanishing if and only if the first Chern class of $Y$ vanishes, i.e., if and only if $Y$ is trivial. 
Let us now generalise this result to $\mathbb{T}^{n}$-principal bundles. For this, we define

$$
\mathbb{T}_{i}^{n}=\left\{\left(z_{1}, \ldots, z_{n}\right) \in \mathbb{T} \text { such that } z_{i}=1\right\}
$$

and we let $Y_{i}=Y / \mathbb{T}_{i}^{n}$ denote the quotient space for the action of $\mathbb{T}_{i}^{n}$ on a principal $\mathbb{T}^{n}$-bundle $Y \rightarrow X$. Then $Y_{i}$ is a $\mathbb{T}$-principal bundle with action induced by the inclusion $\alpha_{i}: \mathbb{T} \hookrightarrow \mathbb{T}^{n}$ of the $i$-th factor and with base space $X$. Moreover $Y$ is isomorphic as a $\mathbb{T}^{n}$-bundle to $\alpha_{1}^{*} Y_{1} * \cdots \alpha_{n}^{*} Y_{n}$, where $\alpha_{i}^{*} Y_{i}$ is the $\mathbb{T}^{n}$-bundle induced from $Y_{i}$ by $\alpha_{i}$. Consequently $Y$ is completely determined (up to isomorphism of $\mathbb{T}^{n}$-bundle) by the first Chern classes of the principal $\mathbb{T}$-bundles $Y_{i}$, and $Y$ is a trivial $\mathbb{T}^{n}$-bundle if and only if all the $Y_{i}$ are trivial.

Proposition 5.5. Let $Y \stackrel{q}{\rightarrow} X$ be a principal $\mathbb{T}^{n}$-bundle. Then the $(0,1)$-degree component of the differential

$$
d_{2}^{0,1}: H^{0}\left(X, \mathrm{~K}^{1}\left(Y_{x}\right)\right) \rightarrow H^{2}\left(X, \mathrm{~K}^{0}\left(Y_{x}\right)\right)
$$

on $E_{2}^{*, *}(Y)$ vanishes if and only if $Y \stackrel{q}{\rightarrow} X$ is trivial.

Proof. Since $\mathrm{K}^{*}(Y)$ is equipped with an algebra structure, $d_{2}$ is a map of differential algebra. The unital algebra $\mathrm{K}^{*}\left(Y_{x}\right)$ being generated by the image of $\mathrm{K}^{*}\left(Y_{i, x}\right)$ under the morphism induced by the projection map $Y \rightarrow Y_{i}$, the map $d_{2}^{0,1}$ is completely determined by the image of elements coming from $\mathrm{K}^{*}\left(Y_{i, x}\right)$. The projection map $Y \rightarrow Y_{i}$ provides a morphism of spectral sequences $\left(E_{r}^{p, q}\left(Y_{i}\right), d_{r}\right) \rightarrow\left(E_{r}^{p, q}(Y), d_{r}\right)$. In particular, we obtain a commutative diagram

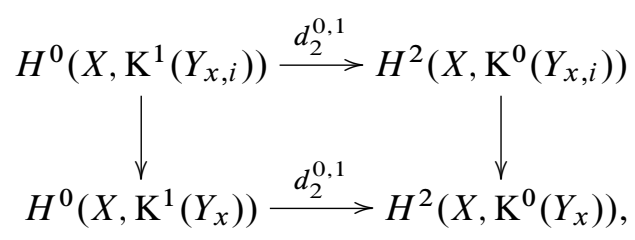

where the vertical arrows are induced by the projection of the fiber $\pi_{i}: Y_{x} \rightarrow Y_{i, x}$. Since the inclusion $\mathrm{K}^{0}\left(Y_{i, x}\right) \rightarrow \mathrm{K}^{0}\left(Y_{x}\right)$ is injective (since it sends [1] to [1]) the right vertical map is injective, too. Thus it follows from Proposition 5.3 that the range of the left vertical map of the diagram lies in the kernel of $d_{2}^{0,1}: H^{0}\left(X, \mathrm{~K}^{1}\left(Y_{x}\right)\right) \rightarrow$ $H^{2}\left(X, \mathrm{~K}^{0}\left(Y_{x}\right)\right)$ if and only if $Y_{i}$ is trivial.

Remark 5.6. (1) If $Y \cong X \times \mathbb{T}^{n}$ is the trivial $\mathbb{T}^{n}$-bundle over $X$, then $d_{2}: E_{2}^{*, *} \rightarrow E_{2}^{*, *}$ vanishes completely. To see this, we can use the Künneth formula in K-theory to show that it is enough to prove the result for the Leray-Serre spectral sequence associated with $C_{0}(X)$, i.e., the Hirzebruch spectral sequence for the K-theory of $X$. Then $E_{2}^{p, q}(X)=H^{p}(X, \mathbb{Z})$ if $p-q$ is even and $E_{2}^{p, q}(X)=0$ 
if $p-q$ is odd. Since $d_{2}$ maps $E_{2}^{p, q}(X)$ to $E_{2}^{p+2, q+1}(X)$, it follows that either the source or the target of this map must be zero. Thus $d_{2}=0$. As a direct consequence of this observation and of Proposition 5.5 we now see that a principal $\mathbb{T}^{n}$-bundle $Y \stackrel{q}{\rightarrow} X$ is trivial if and only if all $d_{2}$-differentials in the associated spectral sequence vanish.

(2) More generally, if $Y \stackrel{q}{\rightarrow} X$ is a principal $\mathbb{T}^{n}$-bundle, then $\mathrm{K}^{*}(Y)$ is endowed with a $\mathrm{K}^{*}(X)$-module structure. Therefore $\bigoplus E_{2}^{p, q}(Y)=\bigoplus H^{p}\left(X, \mathrm{~K}^{p-q}\left(Y_{x}\right)\right)$ has a $\bigoplus H^{p}\left(X, \mathrm{~K}^{p-q}(\{*\})\right)$-module structure provided by the cup product. Thus, $\bigoplus H^{0}\left(X, \mathrm{~K}^{p-q}\left(Y_{x}\right)\right)$ being a generating set and since the differential $d_{2}$ is $\bigoplus H^{p}\left(X, \mathrm{~K}^{p-q}(\{*\})\right)$-linear, $d_{2}$ is completely determined by the Chern classes $c_{i}$.

Proof of Theorem 5.2. Using the fact that $\mathcal{R K K}$-equivalence induces an equivalence of spectral sequences, the result is now a direct consequence of Theorem 5.1 and the above remark.

A natural question is then: Let $A(X)$ be a NCP $\mathbb{T}^{n}$-bundle with classifying data $([Y], f)$. Can we recover any information concerning $Y$ from the spectral sequence $\left\{E_{r}^{* * *}(A), d_{r}\right\}$ derived from the $\mathrm{K}_{*}$-fibration $A(X)$ ? As we shall see below, this is not always the case.

With the above notation, let $q_{i}: Y \rightarrow Y_{i}=Y / \mathbb{T}_{i}^{n}$ denote the quotient map. Let us denote by $U_{i}, W_{i j}$ the images of the generators $g_{i}, f_{i j} \in H_{n}$ in the group $\mathrm{C}^{*_{-}}$ algebra $C^{*}\left(H_{n}\right)$. There are canonical $C_{0}(X)$-linear*-homomorphisms $\Lambda_{i}: C\left(Y_{i}\right) \rightarrow$ $A(X)=Y * f^{*}\left(C^{*}(H)\right)(X)$ given in the following way: First let us define a *homomorphism $\tilde{\Lambda}_{i}: C_{0}\left(Y_{i}\right) \rightarrow\left(C_{0}(Y) \otimes C(\mathbb{T})\right)^{\mathbb{T}^{n}}$ by $\left(\tilde{\Lambda}_{i}(\phi)\right)(y, t)=\phi\left(t q_{i}(y)\right)$, where $\mathbb{T}$ is acted upon by $\mathbb{T}^{n}$ using the projection on the $i$-th component. Next we identify $C\left(\mathbb{T}_{i}\right)$ with $C^{*}\left(U_{i}\right)$ via functional calculus to obtain from this a welldefined $C_{0}(X)$-linear *-homomorphism (also called $\left.\tilde{\Lambda}_{i}\right)$ from $C_{0}\left(Y_{i}\right)$ to $\left(C_{0}(Y) \otimes\right.$ $\left.C^{*}\left(U_{i}\right)\right)^{\mathbb{T}^{n}}$. Because $U_{i}$ commutes with all $W_{k j}$, the $\mathrm{C}^{*}$-algebra $C^{*}\left\langle U_{i}, W_{k j} ; 1 \leq\right.$ $k<j \leq n\rangle$ is a $C\left(\mathbb{T}^{n(n-1) / 2}\right)$-subalgebra of $C^{*}\left(H_{n}\right)$ isomorphic to $C\left(\mathbb{T}^{n(n-1) / 2}\right) \otimes$ $C^{*}\left(U_{i}\right)$ and hence, as $C_{0}(X)$-algebras, we can identify $\left(C_{0}(Y) \otimes C^{*}\left(U_{i}\right)\right)^{\mathbb{T}^{n}} \cong$ $\left.\left(C_{0}(Y) \otimes C_{0}(X) C\left(\mathbb{T}^{n(n-1) / 2}\right) \otimes C^{*}\left(U_{i}\right)\right)\right)^{\mathbb{T}^{n}}$ with a subalgebra of $Y * f^{*}\left(C^{*}\left(H_{n}\right)\right)=$ $\left(C_{0}(Y) \otimes C_{0}(X) f^{*} C^{*}\left(H_{n}\right)\right)^{\mathbb{T}^{n}}$. The map $\Lambda_{i}$ is then given by the composition of $\tilde{\Lambda}_{i}$ with this inclusion.

The morphism $\Lambda_{i}: C_{0}\left(Y_{i}\right) \rightarrow A(X)$ induces a morphism

$$
\left\{\Lambda_{i, r}^{p, q}: E_{r}^{p, q}\left(Y_{i}\right) \rightarrow E_{r}^{p, q}(A)\right\}
$$

of spectral sequences. At the $E_{2}$-term, the morphism

$$
\Lambda_{i, 2}^{p, q}: H^{p}\left(X, \mathrm{~K}^{p-q}(\mathbb{T})\right) \rightarrow H^{p}\left(X, \mathcal{K}_{p-q}(A)\right)
$$

is induced by the morphism of group bundles

$$
\left(\Lambda_{i, x, *}\right)_{x \in X}: X \times \mathrm{K}^{*}(\mathbb{T}) \rightarrow \mathcal{K}_{*}(A) .
$$


In particular, if $c_{i} \in H^{2}(X, \mathbb{Z})$ is the Chern class of $Y_{i}$, then using the notation of Lemma 5.4 we get

$$
d_{2}^{0,1}\left(\Lambda_{i, 2}^{0,1}\left(\left[\omega_{\nu}\right]\right)=\Lambda_{i, 2}^{2,0}\left(c_{i}\right) .\right.
$$

According to [7], if $X$ is path connected with base point $x$, then the cohomology group $H^{*}\left(X, \mathcal{K}_{*}(A)\right)$ can be described in the following way: Fix a simplicial decomposition of $X$ and lift it to a $\pi_{1}(X)$-invariant simplicial decomposition of $\widetilde{X}$. Let $S_{*}(\tilde{X})$ be the simplicial complex obtained from this simplicial decomposition of $\tilde{X}$. Then $S_{*}(\tilde{X})$ is endowed with an action of $\Gamma=\pi_{1}(X)$ by automorphisms and $H^{*}\left(X, \mathcal{K}_{*}(A)\right)$ is then the cohomology of the complex $\operatorname{Hom}_{\Gamma}\left(\mathcal{S}_{*}(\tilde{X}), \mathrm{K}_{*}\left(A_{x}\right)\right)$ of $\Gamma$-equivariant homomorphisms from $\mathcal{S}_{*}(\tilde{X})$ to $\mathrm{K}_{*}\left(A_{x}\right)$. In particular, in degree zero we get

$$
H^{0}\left(X, \mathcal{K}_{*}(A)\right)=\operatorname{Inv}_{\Gamma} \mathrm{K}_{*}\left(A_{x}\right),
$$

where for an abelian group $N$ equipped with an action of $\Gamma, \operatorname{Inv}_{\Gamma} N$ stands for the set of $\Gamma$-invariant elements of $N$. Since we will need it later on, we can also define at this point the coinvariant for $N$ to be $\operatorname{Coinv}_{\Gamma} N=N /\langle x-\gamma x ; x \in N$ and $\gamma \in \Gamma\rangle$.

Using this, and noticing that the classes $\left\{\left[U_{1, x}\right], \ldots,\left[U_{n, x}\right]\right\}$ of $\mathrm{K}_{1}\left(A_{x}\right)$ are invariant, we get that $\Lambda_{i, 2}^{0,1}\left(\left[\omega_{\nu}\right]\right)=\left[U_{i, x}\right]$ and thus according to eq. (5.2) we finally obtain that $d_{2}^{0,1}\left(\left[U_{i, x}\right]\right)=\Lambda_{i, 2}^{2,0}\left(c_{i}\right)$. Hence we can find the first Chern classes of the $Y_{i}$ in our spectral sequence if and only if $\Lambda_{i, 2}^{2,0}\left(c_{i}\right)$ does not vanish. However, as we shall see below, the map $\Lambda_{i, 2}^{2,0}$ is not injective in general.

The end of the section is devoted to the study of the spectral sequences of NCP $\mathbb{T}^{2}$-bundles with base $\mathbb{T}^{2}$. In case where the underlying function $f: \mathbb{T}^{2} \rightarrow \mathbb{T}$ is homotopic to a constant, we get a complete description by Remark 5.6. If $f$ is not homotopic to a constant, then the only part of the differential $d_{2}$ which does not vanish automatically is

$$
d_{2}^{0,1}: H^{0}\left(\mathbb{T}^{2}, \mathrm{~K}^{1}\left(\mathbb{T}^{2}\right)\right) \rightarrow H^{2}\left(\mathbb{T}^{2}, \mathcal{K}_{0}(A)\right),
$$

since we shall see below that $H^{0}\left(\mathbb{T}^{2}, \mathcal{K}_{0}(A)\right)$ is given by the invariants in $\mathrm{K}_{0}\left(\mathbb{T}^{2}\right)$ under the action of $\mathbb{Z}^{2} \cong \pi\left(\mathbb{T}^{2}\right)$, and hence is generated by the class [1] of the unit. Since this class trivially extends to a class in $\mathrm{K}_{0}(A(X))$, it must vanish under any differential in the spectral sequence.

To proceed let us first remark that if $F:[0,1] \times X \rightarrow \mathbb{T}^{n(n-1) / 2}$ is a homotopy between $f_{0}: X \rightarrow \mathbb{T}^{n(n-1) / 2}$ and $f_{1}: X \rightarrow \mathbb{T}^{n(n-1) / 2}$ and if $q: Y \rightarrow X$ is any $\mathbb{T}^{n}$-bundle, then $(Y \times[0,1]) * F^{*}\left(C^{*}\left(H_{n}\right)\right)$ is a homotopy of NCP $\mathbb{T}^{n}$-bundles and thus according to [10], Proposition 3.2, $Y * f_{0}^{*}\left(C^{*}\left(H_{n}\right)\right)$ and $Y * f_{1}^{*}\left(C^{*}\left(H_{n}\right)\right)$ are $\mathcal{R K K}$-equivalent.

For $X=\mathbb{T}^{2}$, the classifying data are $\left(\left[Y \stackrel{q}{\rightarrow} \mathbb{T}^{2}\right], f\right)$, where $f: \mathbb{T}^{2} \rightarrow \mathbb{T}$ is a continuous function. According to the previous remark, we can replace $f$ by a homotopic function and thus we can assume that there exist integers $k$ and $l$ such that 
$f\left(z_{1}, z_{2}\right)=z_{1}^{k} z_{2}^{l}$ for every $\left(z_{1}, z_{2}\right)$ in $\mathbb{T}^{2}$. Let us compute $H^{*}\left(\mathbb{T}^{2}, \mathcal{K}_{0}(A)\right)$. We have $\pi_{1}\left(\mathbb{T}^{2}\right) \cong \mathbb{Z}^{2}$ with action of the generators $(1,0)$ and $(0,1)$ of $\mathbb{Z}^{2}$ on $\mathrm{K}^{0}\left(\mathbb{T}^{2}\right)$ in the base $([1], \beta)$ given by the matrices $\left(\begin{array}{ll}1 & k \\ 0 & 1\end{array}\right)$ and $\left(\begin{array}{ll}1 & l \\ 0 & 1\end{array}\right)$, respectively (see [10], Proposition 5.2).

Let us fix a simplicial decomposition of $\mathbb{T}^{2}$. Then since $\mathbb{R}^{2} \rightarrow \mathbb{T}^{2}$ is the classifying covering for $\mathbb{Z}^{2}$, the simplicial complex $\delta\left(\mathbb{R}^{2}\right)$ is a free resolution for $\mathbb{Z}\left[\mathbb{Z}^{2}\right]$ and hence for any abelian group $M$ equipped with an action of $\mathbb{Z}^{2}$, the cohomology of the complex $\operatorname{Hom}_{\mathbb{Z}^{2}}\left(S\left(\mathbb{R}^{2}\right), M\right)$ is naturally isomorphic to $H^{*}\left(\mathbb{Z}^{2}, M\right)$. Recall from [13] that for an abelian group $M$ equipped with an action of $\mathbb{Z}^{n}$, the cohomology group $H^{*}\left(\mathbb{Z}^{n}, M\right)$ can be computed recursively in the following way:

- For $n=0$ we have that $H^{0}\left(\mathbb{Z}^{n}, M\right) \cong M$ and $H^{k}\left(\mathbb{Z}^{n}, M\right)=\{0\}$ for $k \geq 1$.

- Let us consider the action of $\mathbb{Z}^{n-1}$ on $M$ using the $n-1$ last factors of $\mathbb{Z}^{n}$. Then the action of the first factor of $\mathbb{Z}^{n}$ induces an action of $\mathbb{Z}$ on $H^{k}\left(\mathbb{Z}^{n-1}, M\right)$ and there is an natural exact sequence

$$
\left.0 \rightarrow \operatorname{Coinv}_{\mathbb{Z}} H^{k}\left(\mathbb{Z}^{n-1}, M\right) \rightarrow H^{k}\left(\mathbb{Z}^{n}, M\right) \rightarrow \operatorname{Inv}_{\mathbb{Z}} H^{k-1}\left(\mathbb{Z}^{n-1}, M\right)\right) \rightarrow 0 .
$$

From this, it is straightforward to check that $H^{n}\left(\mathbb{Z}^{n}, M\right)$ is naturally isomorphic to $\operatorname{Coinv}_{\mathbb{Z}^{n}} M$. In the case $M=\mathbb{Z}$ equipped with the trivial action of $\mathbb{Z}^{n}$, the corresponding identification $H^{n}\left(\mathbb{Z}^{n}, \mathbb{Z}\right) \cong \operatorname{Coinv}_{\mathbb{Z}^{n}} \mathbb{Z} \cong \mathbb{Z}$ is given by pairing with the fundamental class of $H_{n}\left(\mathbb{Z}^{n}, \mathbb{Z}\right)$. Under the natural identification $H_{*}\left(\mathbb{Z}^{n}, \mathbb{Z}\right) \cong$ $H_{*}\left(\mathbb{T}^{n}, \mathbb{Z}\right)$, this class can be viewed as the fundamental class $\left[\mathbb{T}^{n}\right]$ of $H_{n}\left(\mathbb{T}^{n}, \mathbb{Z}\right)$.

Combining all this, we are now in the position to describe the $d_{2}$ map of the spectral sequence derived from a NCP $\mathbb{T}^{2}$-bundle $A\left(\mathbb{T}^{2}\right)$ with classifying data $\left(\left[Y \stackrel{q}{\rightarrow} \mathbb{T}^{2}\right], f\right)$. Let $k$ be the greatest common divisor of the winding numbers of the two components of $f$. We can assume that $k \neq 0$, otherwise $f$ is homotopic to a constant map and thus $A\left(\mathbb{T}^{2}\right)$ is $\mathcal{R K K}$-equivalent to $C(Y)$. Then we have:

- $H^{2}\left(\mathbb{T}^{2}, \mathcal{K}_{0}(A)\right) \cong \operatorname{Coinv}_{\mathbb{Z}^{2}} \mathrm{~K}^{0}\left(\mathbb{T}^{2}\right) \cong \mathbb{Z} / k \mathbb{Z} \oplus \mathbb{Z}$, where the image in $\operatorname{Coinv}_{\mathbb{Z}^{2}} \mathrm{~K}_{0}\left(\mathbb{T}^{2}\right)$ of the class [1] $\in \mathrm{K}_{0}\left(\mathbb{T}^{2}\right)$ is a generator for $\mathbb{Z} / k \mathbb{Z}$, and where the image of the Bott element $\beta \in \mathrm{K}_{0}\left(\mathbb{T}^{2}\right)$ is a generator for $\mathbb{Z}$.

- Up to this identification, $d_{2}^{0,1}$ has range in $\mathbb{Z} / k \mathbb{Z}$ and

$$
d_{2}^{0,1}\left(\left[U_{i, z}\right]\right)=\left\langle c_{i},\left[\mathbb{\nabla}^{2}\right]\right\rangle \bmod k
$$

for $z=f(1,1), i=1,2$, and where $c_{i}$ is the Chern class of the $\mathbb{T}$-bundle $Y / \mathbb{T}_{i}^{2} \rightarrow X$

Remark 5.7. (1) In particular, for the function $f\left(z_{1}, z_{2}\right)=z_{1}$, the $d_{2}$ map vanishes for any principal $\mathbb{T}^{2}$-bundle $Y \rightarrow \mathbb{T}^{2}$. We actually do not know at this stage whether all bundles corresponding to the function $f\left(z_{1}, z_{2}\right)=z_{1}$ are $\mathcal{R K K}$-equivalent, so we cannot answer the general question whether two NCP bundles with isomorphic 
spectral sequences must be $\mathcal{R K K}$-equivalent. We plan to investigate this question in future work.

(2) The above computation can be carried out for any compact oriented surface $X$. The reason is that the fundamental group

$$
\Gamma=\left\langle a_{i}, b_{i}: i=1, \ldots n ; \prod_{i=1, \ldots, n}\left[a_{i}, b_{i}\right]=1\right\rangle
$$

(where $n$ is the genus of $X$ ) of such surface satisfies Poincaré duality and thus

$$
H^{2}\left(X, \mathcal{K}_{0}(A)\right) \cong \operatorname{Coinv}_{\Gamma} \mathrm{K}_{0}\left(A_{x}\right) \cong \mathbb{Z} / k \mathbb{Z} \oplus \mathbb{Z}
$$

where $k$ is the greatest common divisor of $\left\langle f, a_{i}\right\rangle,\left\langle f, b_{i}\right\rangle, i=1, \ldots, n$, and for any $\gamma$ in $\Gamma$, the integer $\langle f, \gamma\rangle$ is the winding number of $f \circ h$ for a map $h: \mathbb{T} \rightarrow X$ representing the element $\gamma$.

\section{References}

[1] P. Baum, A. Connes, and N. Higson, Classifying space for proper actions and $K$-theory of group $C^{*}$-algebras. In $C^{*}$-algebras: 1943-1993 (San Antonio, TX, 1993), Contemp. Math. 167, Amer. Math. Soc., Providence, RI, 1994, 240-291. Zbl 0830.46061 MR 1292018

[2] B. Blackadar, K-theory for operator algebras. Math. Sci. Res. Inst. Publ. 5, 2nd ed., Cambridge University Press, Cambridge 1998. Zbl 0913.46054 MR 1656031

[3] J. Chabert, S. Echterhoff, and R. Nest, The Connes-Kasparov conjecture for almost connected groups and for linear $p$-adic groups. Publ. Math. Inst. Hautes Études Sci. (2003), 239-278. Zbl 1048.46057 MR 2010742

[4] J. Chabert, S. Echterhoff, and H. Oyono-Oyono, Going-down functors, the Künneth formula, and the Baum-Connes conjecture. Geom. Funct. Anal. 14 (2004), 491-528. Zbl 1063.46056 MR 2100669

[5] J. Cuntz, $K$-theory and $C^{*}$-algebras. In Algebraic $K$-theory, number theory, geometry and analysis (Bielefeld, 1982), Lecture Notes in Math. 1046, Springer, Berlin 1984, 55-79. Zbl 0548.46056 MR 0750677

[6] M. Dadarlat, Fiberwise $K K$-equivalence of continuous fields of $\mathrm{C}^{*}$-algebras. $J$. $K$-theory 3 (2009), 205-219.

[7] J. F. Davis and P. Kirk, Lecture notes in algebraic topology. Grad. Stud. Math. 35, Amer. Math. Soc., Providence, RI, 2001. Zbl 1018.55001 MR 1841974

[8] E. Dyer, Cohomology theories. Mathematics Lecture Note Series, W. A. Benjamin, Inc., New York 1969. Zbl 0182.57002 MR 0268883

[9] S. Echterhoff, W. Lück, C. Phillips, and S. Walters. The structure of crossed products of irrational rotation algebras by finite subgroups of $\mathrm{SL}_{2}(\mathbb{Z})$. To appear in J. Reine Angew. Math. 
[10] S. Echterhoff, R. Nest, and H. Oyono-Oyono, Principal noncommutative torus bundles. Proc. London Math. Soc., Advance Access published November 25, 2008, doi:10.1112/plms/pdn050.

[11] S. Echterhoff and D. P. Williams, Crossed products by $C_{0}(X)$-actions. J. Funct. Anal. 158 (1998), 113-151. Zbl 0909.46055 MR 1641562

[12] S. Echterhoff and D. P. Williams, Locally inner actions on $C_{0}(X)$-algebras. J. Operator Theory 45 (2001), 131-160. Zbl 0994.46023 MR 1823065

[13] A. H. Forrest and J. Hunton, The cohomology and $K$-theory of commuting homeomorphisms of the Cantor set. Ergodic Theory Dynam. Systems 19 (1999), 611-625. Zbl 0954.54020 MR 1695911

[14] N. Higson and G. Kasparov, $E$-theory and $K K$-theory for groups which act properly and isometrically on Hilbert space. Invent. Math. 144 (2001), 23-74. Zbl 0988.19003 MR 1821144

[15] N. Higson, V. Lafforgue, and G. Skandalis, Counterexamples to the Baum-Connes conjecture. Geom. Funct. Anal. 12 (2002), 330-354. Zbl 1014.46043 MR 1911663

[16] D. Husemoller, Fibre bundles. Grad. Texts in Math. 20, 3rd ed., Springer-Verlag, New York 1994. Zbl 0794.55001 MR 1249482

[17] G. G. Kasparov, Equivariant $K K$-theory and the Novikov conjecture. Invent. Math. 91 (1988), 147-201. Zbl 0647.46053 MR 918241

[18] E. Kirchberg and S. Wassermann, Permanence properties of $C^{*}$-exact groups. Doc. Math. 4 (1999), 513-558. Zbl 0958.46036 MR 1725812

[19] J. McCleary, A user's guide to spectral sequences. Cambridge Stud. Adv. Math. 58, 2nd ed., Cambridge University Press, Cambridge 2001. Zbl 0959.55001 MR 1793722

[20] R. Meyer and R. Nest, The Baum-Connes conjecture via localisation of categories. Topology 45 (2006), 209-259. Zbl 1092.19004 MR 2193334

[21] J. L. Tu, La conjecture de Novikov pour les feuilletages hyperboliques. K-Theory 16 (1999), 129-184. Zbl 0932.19005 MR 1671260

[22] J.-L. Tu, La conjecture de Baum-Connes pour les feuilletages moyennables. $K$-Theory 17 (1999), 215-264. Zbl 0939.19001 MR 1703305

[23] D. P. Williams, The structure of crossed products by smooth actions. J. Austral. Math. Soc. Ser. A 47 (1989), 226-235. Zbl 0687.46044 MR 1008836

Received October 1, 2008

S. Echterhoff, Westfälische Wilhelms-Universität Münster, Mathematisches Institut,

Einsteinstr. 62, 48149 Münster, Germany

E-mail: echters@uni-muenster.de

R. Nest, Department of Mathematics, University of Copenhagen, Universitetsparken 5, 2100 Copenhagen, Denmark

E-mail: rnest@math.ku.dk

H. Oyono-Oyono, Université Blaise Pascal de Clermont-Ferrand, Laboratoire de Mathématiques, Plateau des Cézeaux, 63177 Aubière Cedex, France

E-mail: oyono@math.cnrs.fr 